Revue musicale OICRM

revue musicale oicrm

\title{
Masques, op. 34 de Karol Szymanowski. Une lecture architecturelle des principes harmoniques de « Tantris le bouffon ", et esquisse d'une trajectoire interprétative
}

\section{Christophe Alvarez}

Volume 4, numéro 1, 2017

URI : https://id.erudit.org/iderudit/1040302ar

DOI : https://doi.org/10.7202/1040302ar

Aller au sommaire du numéro

Éditeur(s)

Observatoire interdisciplinaire de création et recherche en musique (OICRM)

ISSN

2368-7061 (numérique)

Découvrir la revue

Citer cet article

Alvarez, C. (2017). Masques, op. 34 de Karol Szymanowski. Une lecture architecturelle des principes harmoniques de "Tantris le bouffon ", et esquisse d'une trajectoire interprétative. Revue musicale OICRM, 4(1), 108-149. https://doi.org/10.7202/1040302ar
Résumé de l'article

L'objectif de cette étude est de montrer - par une analyse rigoureuse des principes harmoniques qui gouvernent les multiples sections de la pièce qu'au-delà d'une apparente hétérogénéité de surface, le discours musical de " Tantris le bouffon » des Masques, op. 34 de Karol Szymanowski est organisé de façon cohérente et subtile par l'interpénétration du niveau de perception immédiate du corps sonore et du niveau infra-sensible qui procède de l'ordre des fondamentales harmoniques. Cet article propose également des conseils d’interprétation, déterminés par l'expérience analytique. 


\title{
Masques, op. 34 de Karol Szymanowski. Une lecture architecturelle des principes harmoniques de « Tantris le bouffon ", et esquisse d'une trajectoire interprétative
}

\author{
Christophe Alvarez
}

Résumé

L'objectif de cette étude est de montrer - par une analyse rigoureuse des principes harmoniques qui gouvernent les multiples sections de la pièce - qu'au-delà d'une apparente hétérogénéité de surface, le discours musical de "Tantris le bouffon » des Masques, op. 34 de Karol Szymanowski est organisé de façon cohérente et subtile par l'interpénétration du niveau de perception immédiate du corps sonore et du niveau infrasensible qui procède de l'ordre des fondamentales harmoniques. Cet article propose également des conseils d'interprétation, déterminés par l'expérience analytique.

Mots clés : conception formelle; harmonie ; interprétation ; organisation quintale ; pivots.

\section{Abstract}

The purpose of this study is to show-by means of a rigorous analysis of the harmonic principles which govern the multiple sections of the work - that beyond the apparently heterogeneous surface the musical discourse of "Tantris the Clown" from the Masks, op. 34 by Karol Szymanowski is subtly and coherently organised by the interpenetration of two layers: the immediate perception of sound structure and the underlayer which proceeds from the order of the harmonic fundamentals. This article also proposes suggestions for interpretation, determined by the analytical experience.

Keywords: formal conception; harmony; interpretation; pillars; quintal organization. 
INTRODUCTION

Karol Szymanowski est né en 1882 à Tymoszówka (actuellement Tymochivka, en Ukraine), petit village sous gouvernorat de Kiev au sein de l'Empire russe. Considéré par les polonais comme l'héritier de Frédéric Chopin, il incarne aussi la figure du renouveau de la musique polonaise du Xxe siècle. Au cours des années 1910, s'émancipant hors de l'influence prégnante des compositeurs germaniques postromantiques, Szymanowski s'est aventuré dans la voie d'une modernité musicale qu'il avait pu découvrir lors de récents séjours en France - séjours qui lui avaient permis d'entrer en contact avec Debussy, Stravinsky et Ravel. S'assimilant les techniques de composition de ces derniers, Szymanowski élabore à cette période une écriture tout à fait originale. Sans abandonner totalement la référence à l'idiome tonal, sa poétique se développe comme une synthèse de plusieurs univers musicaux de son époque : celui qu'il est convenu d'appeler l'" impressionnisme " musical - caractérisé par l'emploi (au lieu de la simple gamme majeure/mineure du système tonal) d'une riche diversité d'échelles de sons aux propriétés remarquables (l'échelle par tons entiers, l'échelle octotonique, etc.) -, mais aussi celui du russe Scriabine, chez qui le chromatisme hérité de Wagner joue un rôle central.

Nous organisons cet article en deux parties : tout d'abord un état des lieux scientifique de la littérature consacrée aux Masques, op. $34^{1}$, suivi d'une clarification méthodologique et de la démarche, puis l'analyse de la pièce centrale du triptyque «Tantris le bouffon ", certainement la plus difficile d'accès du point de vue de la complexité harmonique dont elle fait preuve. L'analyse invoque le rôle joué par les éléments de dynamique, d'accentuel, d'agogique, de métrique, au sein de complexes sonores qui s'impliquent, de façon subtile, dans la structure de l'œuvre. Elle aborde également la gestuelle pianistique nécessaire à l'accomplissement technique d'instants clés du discours musical et au surlignage, dans des espaces texturels fournis, du matériau signifiant en terme d'élaboration formelle.

Si cette étude laisse délibérément de côté la question de l'affirmation d'un programme musical à travers la prégnance d'influences littéraires ou picturales qui ont pu présider à la détermination des titres des pièces du triptyque Masques $^{2}$, elle s'attache en revanche à éclairer la construction architecturale de l'œuvre par l'analyse et le relevé de systèmes de structuration du discours par l'intermédiaire d'une étude harmonique basée pour sa majeure partie sur l'expressivité sonore des agrégats, et par l'usage d'outils théoriques développés au cours des années 1980 dans la musicologie

1 Le triptyque Masques, op. 34 (1915) fait partie d'une série d'œuvres écrites entre 1914 et 1916 (Chants d'amour de Hafiz, op. 26, 1914 ; Métopes, op. 29, 1915 ; Douze études, op. 33, 1916 ; Symphonie no 3, op. 27, 1916) qui marquent une véritable rupture esthétique avec les œuvres qui les précèdent. Il s'agit là d'une profonde remise en question artistique et de la réévaluation complète des techniques d'écritures et de l'usage du matériau musical. La partition utilisée et à partir de laquelle sont tirés les exemples musicaux est celle publiée par Universal Edition en 1919. Elle appartient désormais au domaine public et peut être consultée ici : http://imslp.org/wiki/3 Masques, Op.34 (Szymanowski,_Karol) (consulté le 12 octobre 2016).

2 Les trois pièces du recueil portent les titres suivants : "Shéhérazade ", "Tantris le bouffon ", «Sérénade de Don Juan ». 
anglo-saxonne et théorisés notamment par George Perle et Allen Forte. Néanmoins, et afin d'éviter l'écueil d'une démarche analytique empirique, aucune méthode préétablie n'est invoquée ; la méthodologie que nous construisons ici est fonction de la topographie du discours et des éléments (motiviques, harmoniques, ornementaux) saillants qui apparaissent tout d'abord lors de l'expérience auditive et qui dirigent alors l'analyste dans un cheminement de découvertes progressives d'une construction formelle à plusieurs niveaux de lecture.

L'objectif de cette étude est de dégager un principe organisateur qui, sous-tendant le flux musical, est à même de déterminer les caractéristiques de progression harmonique de ce dernier ainsi que, dans une certaine mesure, son parcours mélodique. Il s'agit de mettre en lumière le liant qui permet d'unifier l'apparente hétérogénéité de surface en un ensemble cohérent au sein duquel l'enchaînement des différentes sections et la physionomie harmonique des complexes sonores obéissent à un schéma structurel défini. La révélation d'une substance organisatrice du discours donne également l'opportunité à l'interprète de porter un regard neuf sur l'œuvre et ses implications, et de souligner en conséquence les instants musicaux spécifiques qui produiront, chez l'auditeur, l'image sonore d'un espace ordonné et stabilisé selon des principes clarifiés.

\section{LITTÉRATURE SCIENTIFIQUE EXISTANTE}

Il n'existe que peu d'ouvrages ou études ayant effectué une démarche analytique conséquente du discours musical des Masques, op. 34 de Karol Szymanowski. Les deux principaux sont les thèses de doctorat rédigées par Marylynn Louise Fletcher (1984) et Donald Zent (1988). Signalons également l'existence de deux autres documents qui se concentrent davantage sur l'implication du corpus littéraire ayant déterminé les titres du triptyque qu'au détail analytique du discours, par la réalisation d'une exégèse programmatique qui place au premier plan des suppositions d'ordre subjectif sur le signifiant dramaturgique de l'espace musical, et relègue au second plan les faits tangibles de la réalité sonore ; il s'agit des études publiées par Awakawa-Imai Tchié (2009) et Cesetti Durval (2012). Ce dernier procède à la différenciation de deux notions esthétiques qu'il nomme "statisme et mouvement " et les associe à deux grands archétypiques du discours harmonique : l'un se fondant sur un "but tonal ", l'autre se détachant totalement de cet idiome dans une conception toute " impressionniste ». Ces notions d'immobilisme/directionnalité, liées également à la dualité impressionnisme/expressionnisme et plus largement à l'opposition consonance/dissonance qui incarne la grande problématique de la transition post-tonale, ont été largement développées par Ernst Kurth, dans son ouvrage sur Tristan de Wagner (1920), qui se positionne par rapport aux théories développées par Stumpf $(\underline{1898} ; \underline{1911})$ et Riemann (1901) sur le sujet. Or, Cesetti associe la notion de mouvement à des instants du discours qu'il aborde à travers le prisme de la tonalité et les attractions dominante/tonique qu'il y aperçoit, tandis qu'il considère les complexes harmoniques altérés et l'imbrication des lignes texturelles (qui constituent, soulignons-le, la plus grande part du mode d'écriture des Masques) comme des « filigranes superficiels » desquels se dégage en fait une "immobilité essentielle ».

Parmi les ouvrages consacrés à d'autres œuvres de Szymanowski, citons un article très intéressant publié par Ann K. McNamee (1985) dans le Journal of Music 
Theory et consacré à l'analyse des Mazurkas, op. 50. L'auteure développe sa théorie autour du cycle des quintes qu'elle voit agir comme élément structurant tant dans la surface du discours immédiatement perceptible (intervalles de quinte déployés à la basse, notamment), que dans l'organisation des rapports harmoniques entre eux et le déploiement des lignes mélodiques. Elle associe le cycle des quintes à l'agencement intervallique du mode acoustique, prenant comme point de départ à sa démonstration le segment pentatonique non altéré qu'elle dispose comme une succession de quatre quintes. Elle invoque également le rôle du double triton (tétracorde 4-25) qui peut participer de la création de deux accords de qualité dominante, dont aucun cependant n'affirme le I $^{\text {er }}$ degré du mode, marquant bien le statut incertain de ce mode vis-à-vis de l'espace tonal. Elle omet néanmoins de signaler la capacité du mode acoustique à exprimer le mode mineur mélodique ascendant lorsque la cinquième note devient $\mathrm{I}^{\mathrm{er}}$ degré, impliquant de ce fait l'accord de neuvième fondé sur la deuxième note comme $v^{e}$ degré de qualité dominante. On voit bien alors le rôle complexe joué par la première note du mode, soit en tant qu'unité indépendante se suffisant à elle-même, soit prise au sein d'un geste rappelant l'idiome tonal et devenant de fait $\mathrm{IV}^{\mathrm{e}}$ degré.

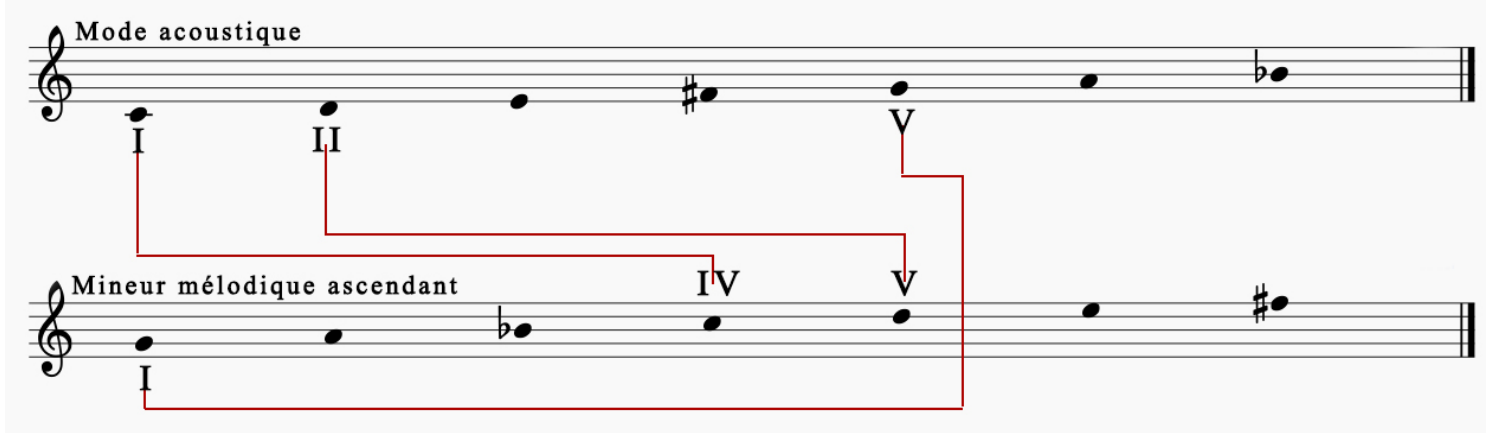

Figure 1 : Relations de degrés entre le mode acoustique et le mode mineur mélodique ascendant.

Si la démarche de McNamee est particulièrement remarquable, c'est qu'elle cherche à réunir la survenance d'éléments multiples et à première vue hétéroclites au sein d'une explicitation théorique qui permet de mettre à jour une trajectoire architecturelle dans l'œuvre. En effet, les compositeurs du début du xx siècle désireux, comme leurs prédécesseurs, d'unifier leurs œuvres par l'intermédiaire de signifiants, usent de différentes formes dans la mise en œuvre de cette unification : à la surface du discours par des relations d'ordre mélodique assimilées à des espaces motiviques (réminiscence d'une forme de construction thématique classique), mais également - et parfois dans le même temps - de façon plus abstraite à travers des cellules intervalliques qui procèdent tant du dessin mélodique que du parcours harmonique ${ }^{3}$.

3 Les Masques présentent, à ce titre, plusieurs formes mélodiques : la mélodie ornementale libre, décrivant un espace improvisé sans implication structurelle ; la mélodie ornementale subordonnée, exprimant un signifiant harmonique lié à la structure locale ou globale ; la mélodie d'accords, formée par la succession et le déplacement de ceux-ci ; et la mélodie motivique, organisant l'espace musical par ses réitérations, originale par son expression intervallique, mais dont la détermination et les fondements harmoniques participent au bâti de la structure globale. 
Néanmoins, une telle démarche analytique d'unification du discours musical n'avait pas encore été réalisée dans les Masques qui souffrent d'une trop grande superficialité dans leur approche. En effet, des ouvrages les concernant et ci-dessus cités, aucun ne manifeste une révélation des principes d'unicité de l'œuvre, du dénominateur commun autour duquel elle serait à même de se structurer.

Dans sa thèse, Fletcher s'attache à relever dans le discours des groupes de notes qu'elle nomme " cellules » et qu'elle classifie selon le prisme unique de la théorie d'Allen Forte. Or, cet inventaire occulte totalement la dimension harmonique pourtant fondamentale dans la construction texturelle et intrinsèquement stratifiée en plusieurs couches structurelles. L'étude de Zent vise à établir un panorama plus complet de l'organisation du discours du corpus choisi. Néanmoins, la méthode d'analyse harmonique invoquée se trouve en désaccord avec l'explicitation théorique globale de l'ouvrage qui affirme envisager les agrégats comme des unités indépendantes dégagées de toute subordination, en faisant référence à l'apport de l'impressionnisme dans 1'esthétique musicale de Szymanowski. Les accords de qualité dominante, altérés ou non, se voient malheureusement toujours rapportés à leur tonique présumée et se voient rattachés de fait à l'univers tonal ${ }^{4}$. Outre la limitation considérable des possibilités de déduction, cette méthode d'analyse harmonique nie totalement la capacité d'émancipation de l'accord de qualité dominante, qui n'a plus nécessité à se résoudre sur une tonique, et dont le triton fondateur est à présent savouré pour lui-même comme intervalle indépendant (classe d'intervalles 6, CI6).

De plus, malgré sa volonté de mettre à jour la pensée architecturale de Szymanowski, Zent ne parvient pas à dessiner une trajectoire esthétique dans le corpus qu'il choisit (Métopes et Masques), en grande partie parce qu'il omet toujours de considérer le développement harmonique à l'échelle des carrures. Par exemple, aux mesures 40-45 de "Tantris ", la mise en œuvre de cette méthode d'analyse ne parvient pas à révéler le double mouvement quintal décrit par les fondamentales (sib-mib, solb-si), dont toutes forment la basse d'accords de qualité dominante sauf le sol bémol, harmonisé en septième majeure, pôle principal de l'œuvre qui en formera la conclusion. Affirmant également que Szymanowski utilise beaucoup l'agrégat « accord mystique » et ses dérivations, Zent multiplie les exemples musicaux où il souligne son apparition. Toutefois, s'il n'en explicite pas la construction intervallique, il ne le rapproche non plus jamais du mode acoustique dont le tétracorde 4-25 caractéristique du mode figure pourtant au sein de la construction intervallique de l'accord, un élément éminemment distinctif. Or, le mode acoustique, abondamment utilisé par Bartók dans sa musique, est employé tout autant dans le corpus des musiques populaires roumaines de la région des Maramures, que dans la musique goralskie des montagnes Tatras en Pologne où il prend le nom de mode podhalean ${ }^{5}$.

4 Notons qu'il s'agit là d'une tendance que l'on retrouve également dans l'approche de Cesetti lorsqu'il évoque, au sujet du climax de "Shéhérazade " (section meno mosso, mes. 275-281) la répétition de l'accord de neuvième altéré qui « n'est suivi d'aucune résolution » (Durval 2012).

5 La Podhale est une région champêtre au nord de la chaîne montagneuse polonaise des Tatras. 
Ce mode, dont la quarte haussée et la septième abaissée en forment la saillance, est nommé " acoustique " en raison du fait qu'il rassemble les six premiers sons impairs du spectre harmonique. Sa construction intervallique le rend particulièrement équivoque : il peut être entendu, comme c'est souvent le cas chez Debussy, comme un mode de sol altéré (le IV ${ }^{\mathrm{e}}$ degré étant haussé d'un demi-ton), ou comme " un mode composite qui résulte, par interférence, du croisement du mode de sol et $\mathrm{du}$ mode de fa par tritonisation du quatrième degré " (Barraqué 1962, p. 151). On peut aussi le rapporter à une échelle autonome que Perle inventorie sous la forme de 1'ensemble de classes de hauteurs symétrique 7-7 [1,2,4,6/6,8,10,11] (Leleu 2004, note 17, p. 31). En déterminant une interprétation modale de cette dernière, on obtient le mode acoustique en démarrant du degré $4(4,6,8,10,11,1,2)$. Comme nous l'avons déjà souligné plus haut, une autre lecture possible de la même échelle, en partant cette fois du degré $11,(11,1,2,4,6,8,10)$ engendre alors le mode mineur mélodique ascendant associé à l'idiome tonal (ibid.).

\section{AcCORD MYSTIQUe/MODE ACOUSTIQUE}

Nous présentons à présent l'assimilation de l'accord mystique comme participant $\mathrm{du}$ mode acoustique et décrivons, par ses multiples expressions intervalliques, quelques-unes de ses formulations agrégatives à même de générer un modèle pluridimensionnel à visée potentiellement structurante au sein des Masques.

Posons l'accord mystique suivant comme point de départ à notre démonstration :

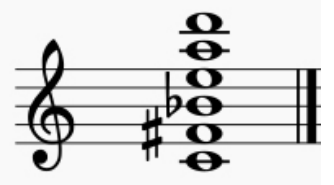

Figure 2: Accord mystique.

Par l'intermédiaire du tétracorde 4-25 présenté par l'agrégat, l'accord mystique a la capacité de générer de façon indépendante trois accords de qualité dominante, c'est-à-dire qu'ils comportent chacun une tierce majeure et une septième mineure par rapport à la tonique : do-mi-fa\#-sib-ré, ré-fa\#-la-sib-do-mi et fa\#-la\#-do-mi.

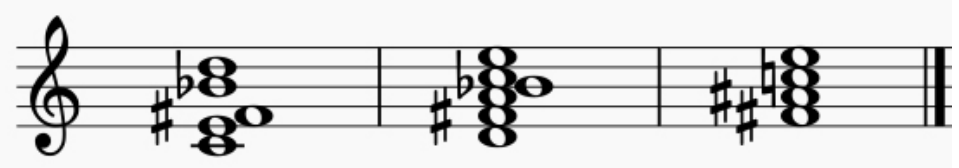

Figure 3 : Trois accords pouvant être formés avec l'accord mystique.

Impliqués au sein du mode acoustique, l'accord sur fa dièse se voit adjoint d'une neuvième mineure (sol), et l'accord sur do d'une quinte fondamentale juste (do-sol). Nous constatons également que ces accords voient tous leur quinte fondamentale altérée : l'accord sur do en présente l'altération descendante, l'accord sur rél'altération ascendante, et l'accord sur fa dièse la double altération. 


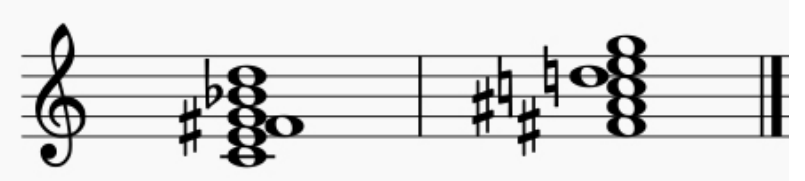

Figure 4: Accords sur do et fa \#, impliqués au sein du mode acoustique.

Dans sa démonstration théorique, Zent n'envisage l'accord mystique que comme une collection de tons entiers (sib-do-ré-mi-fa\#), réduisant de ce fait le champ des interprétations du mode acoustique qui est pourtant considérable. Ce mode participe en effet d'un cycle par tons entiers et par tierces majeures (ré-fa\#-sib), grâce à l'implication du tétracorde 4-25, mais également, découlant de ce même tétracorde, de deux cycles de tierces mineures, mi-sol-sib et $f a \#$-la-do, qui permettent, lorsqu'on leur adjoint la tierce manquante à leur complétion, un remplissage différent de la tierce initiale : do-do\#/réb-mi-fa\#-sol-la-sib ( $3^{1}{ }^{1}$, do\#-mi-sol-sib) ou do-ré-mib-fa\#-sol-la-sib $\left(\mathrm{C} 3^{0}\right.$, do-mib-fa\#-la).

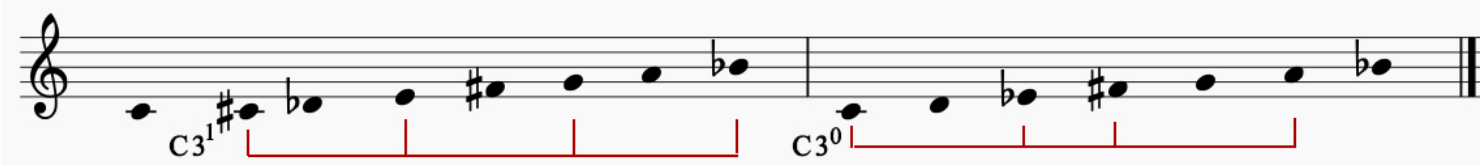

Figure 5 : Génération de deux collections octotoniques par modification de la tierce du mode acoustique.

Les deux subdivisions du triton - considéré comme CI6, c'est-à-dire dégagé de toute implication tonale -, comme suites de tons entiers ou tierces mineures, renvoient aux divisions symétriques de l'octave d'où procèdent - selon le cycle d'intervalles choisi les deux principales échelles à transpositions limitées : l'échelle par tons entiers (C2) et l'échelle octotonique (C3). De ce fait, le triton, par son équivocité intervallique, devient l'intervalle grâce auquel l'interpénétration des échelles se fait le plus naturellement.

On retrouve le tétracorde 4-25 résultant de la combinaison des deux tritons à distance de seconde majeure sib-mi/do-fa\#, à la fois dans l'échelle par tons entiers, dans l'échelle octotonique (où il apparaît, par la superposition des tierces mineures, sous deux formes qui relèvent respectivement de $\mathrm{C} 2^{0}$ et de $\mathrm{C} 2^{1}$ ), et comme nous l'avons vu, dans le mode acoustique. Il dispose également de la capacité de former un accord de septième de dominante avec altération descendante de la quinte ; cet agrégat, issu de l'idiome tonal, tend, lors de sa dissolution, à devenir une unité sonore indépendante considérée à l'aune de sa couleur propre, fruit de ses tensions internes qui procèdent des altérations de la quinte et déterminent une énergie plus directionnelle ou plus statique.

Lors de la complétion du cycle de tierces mineures mi-sol-sib, l'avènement du do dièse dans le mode acoustique permet par exemple la création d'un nouvel accord de qualité dominante fondé sur la: la-do\#-mi-sol-sib. Ajoutée aux trois fondamentales précédemment décrites (do-ré-fa $\#$ ), cette nouvelle fondamentale (la) forme la collection do-ré-fa\#-la, qui reprend les notes constitutives de l'accord de qualité dominante cité plus haut : ré-fa \#-la-do. 


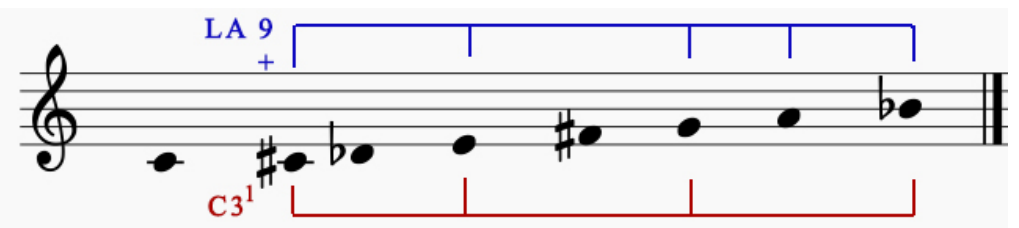

Figure 6: Nouvel accord de qualité dominante sur la généré par la modification de la tierce du mode acoustique.

De ceux potentiellement formés par la disposition de l'accord mystique et intégrés au mode acoustique, les accords fondés sur do et ré sont les plus complets dans le sens où ils présentent à la fois leur quinte fondamentale juste (do-sol et ré-la) et les altérations descendantes et ascendantes de cette quinte, issues des notes altérées du mode acoustique ( $f a \# / s o l b$ et la\#/sib).

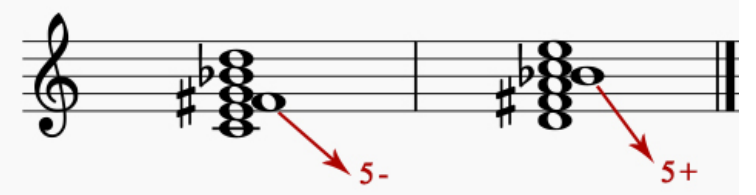

Figure 7 : Deux altérations de la quinte des accords sur do et ré.

Ainsi que nous l'avons dit plus haut, l'imprégnation de l'accord sur fa dièse à l'intérieur du mode acoustique lui permet de s'adjoindre une neuvième mineure. Or, le simple haussement de celle-ci vers la neuvième majeure (sol dièse) qui procèderait d'une généralisation du modèle présenté par les accords sur do et ré (tous deux neuvième majeure), conduirait à l'apparition d'une nouvelle note au sein de la collection qui viendrait compléter l'altération des quintes fondamentales de l'accord sur do (quinte haussée), mais également de l'accord sur ré (quinte abaissée).
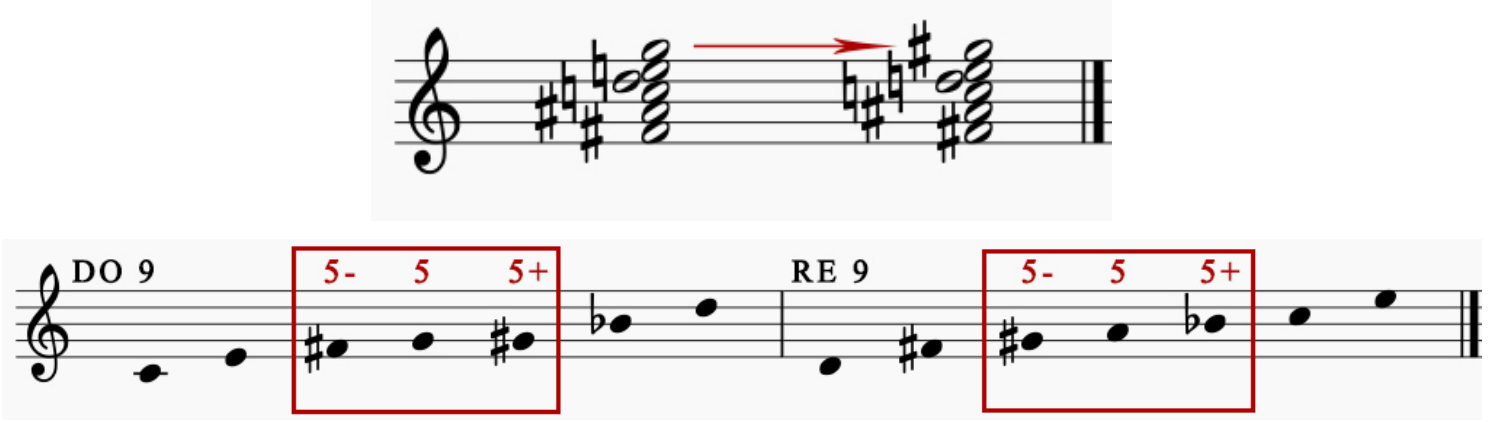

Figure 8: Le haussement à la neuvième majeure (sol\#) permet d'obtenir la double altération de la quinte des accords sur do et ré.

Si le mode acoustique offre la possibilité de former deux accords de neuvième de qualité dominante ${ }^{6}$ et leur double ossature quintale do-sol-ré et ré-la-mi, ce mode nous propose également la double possibilité d'altération de la quinte fondamentale, descendante et ascendante, attachée à une partie du tétracorde 4-25: do-fa\#, ré-sib. 
Le troisième agrégat, découlant de ce même tétracorde, présente, lui, en plus de la double altération de la quinte simultanée, l'altération de la neuvième. De plus, les notes sol-quintes de do et neuvième mineure de fa dièse - et la - quinte de ré - sont les seules barrières au basculement dans l'univers par tons entiers C2 ${ }^{0}$. Celui-ci s'effectuerait par leur jonction sur le sol dièse, note médiante, créant une division en deux segments égaux de l'octave ré-ré. Or, la particularité des notes sol et la est que celles-ci, au centre de la double ossature quintale, provoquent la division d'un nouvel espace clos et symétrique de 14 demi-tons.

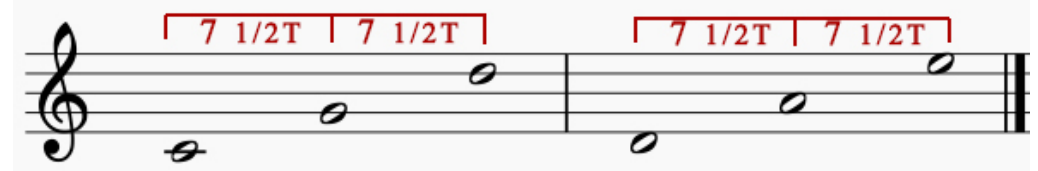

Figure 9: Double ossature quintale et espacement symétrique de sept demi-tons.

À ce titre, les accords de neuvième de qualité dominante sur do et ré sont particulièrement significatifs. Ainsi, leur symétrie quintale repliable autour de la quinte, se complète également d'une symétrie de tierces mineures centrale à son tour repliable autour de la quinte fondamentale, tandis que la double altération de cette quinte forme une gamme par ton complète.

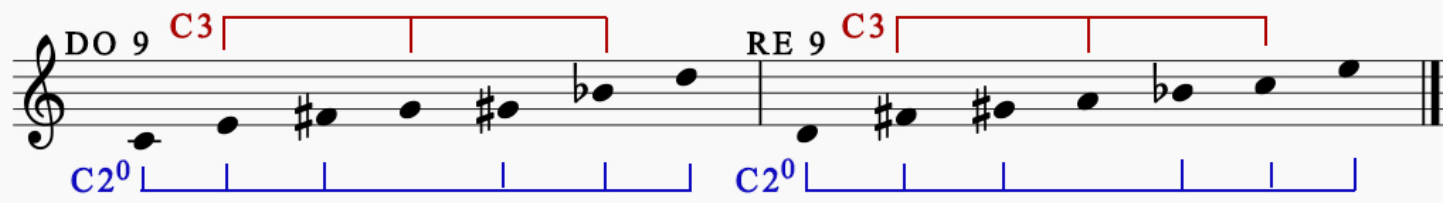

Figure 10: Cycles C3 et C2 formés par l'accord de neuvième altérée de qualité dominante.

Cet accord de neuvième de qualité dominante possède une capacité multiple de se référer tantôt au mode acoustique, à l'échelle par tons entiers, à l'univers tonal à travers son ossature quintale de référence pouvant revêtir, selon l'implication du pôle d'attractivité, les degrés I-II-V ou IV-V-I, à l'échelle octotonique par le cycle de deux tierces mineures qui se voit aisément complété par la minorisation de la neuvième. Nous voyons donc le statut particulier qu'acquiert l'accord de neuvième majeure de qualité dominante contenant précisément toutes les notes résultant de la double subdivision de la CI6 (à la fois un segment de tons entiers et un segment de tierces mineures $)^{7}$.

Nous supposons que cet accord pourrait posséder, au sein du triptyque Masques, la capacité de devenir un modèle d'organisation formelle, tant sur le plan de la surface du discours - c'est-à-dire de déterminer la couleur du déroulement harmonique dans le discours par l'établissement d'une forme d'échelonnage depuis son état naturel jusqu'à la limite de ses possibilités d'altération - que sur le plan infra-sensible - c'est-à-dire 
agissant comme organisateur des rapports intervalliques de la succession des pivots des sections (pôles fondamentaux ou d'attractivité) déterminés par la trajectoire harmonique à l'échelle des carrures ou des cellules motiviques.

\section{ACCORDS DE DOMINANTE}

$\mathrm{Au}$ sujet des accords considérés par 1'harmonie tonale comme accords de dominante - c'est-à-dire ceux ayant, en position fondamentale, à leur sommet, une tierce mineure formant septième avec la basse -, nous entendons ceux-ci comme agrégats indépendants, déterminés par eux-mêmes, et non subordonnés à un geste tonal qui les qualifierait de fait comme précédant une résolution nécessaire. Ainsi, le terme de "qualité dominante " est employé afin de marquer leur distanciation vis-à-vis de l'univers tonal mono-centré, et de signifier leur rôle au sein d'un univers pluridimensionnel où les phénomènes de tension/détente sont réévalués à l'aune du potentiel expressif de chaque accord. L'élargissement de l'accord de septième par l'empilement d'une nouvelle tierce majeure formant neuvième avec la basse est envisagé, dans son état non altéré, en relation directe avec le mode de $s^{8} l^{8}$.

Le pas décisif qu'accomplit ce qu'il est d'usage d'appeler l'impressionnisme musical se situe dans la continuité de l'évolution de la tonalité depuis la période classique qui, durant l'ère romantique, par son élargissement, en était venue à porter en elle, paradoxalement, les germes de sa propre dissolution. Ainsi, l'univers impressionniste considère les accords en tant que blocs sonores, porteurs d'une couleur spécifique, et non plus sous le prisme de la dualité consonance/dissonance. Nous ne sommes plus seulement sensibles au jeu des tensions internes dont vit l'accord ou l'agrégat, et sur lesquelles repose la logique tonale, mais également à une couleur harmonique particulière, qui agit comme un stimulus quasi sensoriel par l'intermédiaire de l'énergie sonore qu'elle porte. Il s'agit là du défi quasi mystique posé par la dissolution de la tonalité, provoquant l'émergence de l'individualité harmonique comme architectonique de la forme, en réaction au système de « masse » induit par la tonalité où les accords se déterminaient par rapport à la volonté d'un centre unique. Au-delà d'une apparente hétérogénéité de surface par la multiplicité des individualités harmoniques, il s'établit un principe unificateur qui agit sur la structure intervallique des accords et qui articule leur succession : les échelles de sons et les cycles d'intervalles qui en découlent.

\section{DÉMARCHE ANALYTIQUE PRÉLIMINAIRE}

Le discours musical des Masques se caractérise par une grande complexité dans sa formulation. Nous y assistons à la fréquente interpénétration des univers se rattachant à

\footnotetext{
8 Ce mode est le seul à pouvoir former, à partir de son $\mathrm{I}^{\mathrm{er}}$ degré, l'ensemble de classes de hauteurs 5-34, exprimant, dans sa disposition en tierces superposées, l'accord de neuvième majeure de dominante issu de l'idiome tonal. La matière sonore n'appelant pas de résolution se trouve considérée pour son expression propre, estimée à l'aune de sa couleur et dont l'énergie, plus statique ou plus directionnelle, est fonction du contexte.
} 
l'octotonisme et aux tons entiers, mais également à certains moments de l'œuvre, nous constatons une certaine forme de subsistance des attractions propres à la tonalité ; de plus, certains instants, nombreux, demeurent à première vue obscurs, car ils ne s'apparentent à aucun des modes de structuration cités ci-dessus. Au travers cette hétérogénéité de surface, les multiples sections, relativement courtes, qui scindent chacune des trois pièces apparaissent comme porteuses d'un signifiant architectural sur lequel nous avons choisi d'organiser notre démonstration. Chaque section manifeste une personnalité texturelle propre. Persuadé que chacune de ces sections agit comme un pilier de soutien pour l'édification de la structure globale, nous nous sommes attaché à en extraire les axes harmoniques autour desquels leur discours s'articule, par le relevé des fondamentales des accords mis en œuvre. La perception de la fondamentale d'un espace agrégatif harmonique défini s'effectue pour moitié par l'expérience auditive, puis par la confirmation textuelle de la hiérarchie qu'occupent les notes : constitutives ou bien étrangères à l'agrégat définissant l'espace harmonique.

Si au cours du premier tiers du Xx ${ }^{\mathrm{e}}$ siècle, de nombreuses œuvres s'articulent autour de principes de "pitch centricity" (" centricité tonale "; Berger 1963), l'organisation de notes autour d'un ou plusieurs pôles de centricité n'inclut pas nécessairement un système hiérarchique de relation à une tonique tel que l'entend la pensée tonale. Néanmoins, à l'aune du relevé topographique des fondamentales, il s'agit de poser la question suivante : ces notes sont-elles toutes d'importance structurelle égale ou bien certaines d'entre elles acquièrent un rôle plus important à même de leur donner la capacité d'agir comme un pôle de centricité pouvant, en quelque sorte, faire graviter un certain nombre de notes autour d'elles?

Ce questionnement va nous conduire à constater la prégnance de certaines notes à même de former une ossature ou un agrégat dont l'implication structurelle au sein du discours sera le point préliminaire à notre démonstration analytique. Plus précisément, il s'agira de deux ossatures de deux quintes chacune, elles-mêmes à distance de quinte. La prégnance des deux pôles de centricité sera confirmée par les rares mais remarquables mouvements cadentiels qui surgissent à des instants clés du discours et qui vont permettre d'évaluer la subsistance d'une certaine forme d'idiome tonal dans lequel le rapport quintal v-I n'est plus envisagé dans une relation de subordination de l'un envers l'autre, mais dans lequel les deux notes sont d'importance égale : l'accord de résolution cesse d'être perçu comme tel lorsque celui-ci se pare à son tour d'une qualité de dominante ${ }^{9}$; la résolution de la dissonance (le triton) vers la consonance (la tierce) se mue en déplacement du triton vers un autre triton à distance de demi-ton ${ }^{10}$, ce geste supprimant de fait, par l'annulation de la consonance, la notion de dissonance elle-même.

9 C'est ce que suggère le mode de sol par la formation d'un accord de neuvième de qualité dominante sur le $\mathrm{I}^{\mathrm{er}}$ degré, déstabilisant de fait le rôle de tonique associé usuellement par l'idiome tonal à ce degré.

10 L'enchaînement décrit ici l'ensemble de classes de hauteurs 4-9, tétracorde miroir tritonique $(0,1,6,7)$, par la capacité qu'il possède également de former deux quintes à distance de triton. 
FONDAMENTALES

Les fondamentales acquièrent un rôle tout particulier dans l'analyse lors de la réalisation du relevé topographique. Nous considérons la fondamentale d'un accord en dehors de toute notion d'attractivité qui se rattacherait à l'univers tonal. À ce titre, la fondamentale d'un accord de qualité dominante est déterminée comme note grave dudit accord en position fondamentale. En aucun cas le terme " fondamentale » utilisé, par exemple, pour un accord de qualité dominante sur sol ne pourra signifier do, mais bien sol.

L'objet de notre étude tend à prouver que les fondamentales forment, dans les Masques, ce que Perle nomme "window of order" ", c'est-à-dire une couche architecturale constituant la cohérence au travers d'un espace relativement chaotique en surface. La construction des accords, bien que se rapportant à des échelles de sons cycliques (tons entiers, tierces mineures), au cycle des quintes (pentatonisme), ou inscrits dans un mode heptatonique, participe, dans son apparente liberté, au développement d'une suggestivité d'ordre impressionniste, au flou des contours. La « window of order " s'incarne par la structuration intrinsèque de l'œuvre qui se détermine par le parcours des fondamentales des sections, fondamentales procédant elles-mêmes de la succession desdits accords. Nous distinguons alors deux niveaux d'organisation qui s'interpénètrent eux-mêmes : un niveau sensible, sur la surface auditivement perceptible du discours, de l'ordre de la couleur des accords et de leur suggestivité ; et un niveau infra-sensible créé par les fondamentales dont le parcours agit comme un modèle des relations harmoniques qui gouvernent le discours musical.

\section{ANALYSE DE LA PIĖCE CHOISIE : «TANTRIS LE BOUFFON »}

Dans les deux dernières mesures qui concluent " Tantris le bouffon ", au-delà de la multiparamétricité harmonique et l'interpénétration au sein du complexe sonore des univers de do et de sol bémol, l'expérience interprétative révèle une dimension auditive nouvelle, d'ordre mélodique, par le timbre que tout pianiste est naturellement enclin à donner aux notes supérieures des accords. Il se dégage ainsi une ligne mélodique de trois notes, constituée d'un demi-ton et d'un ton, et qui, au titre de l'assise fondamentale sur sol bémol présentée à la basse et appuyée par le geste cadentiel de la mesure précédente, semble résonner par la quarte augmentée (do) comme l'esquisse d'un mode de fa sur sol bémol.

11 Voir Perle [1990]1996. 


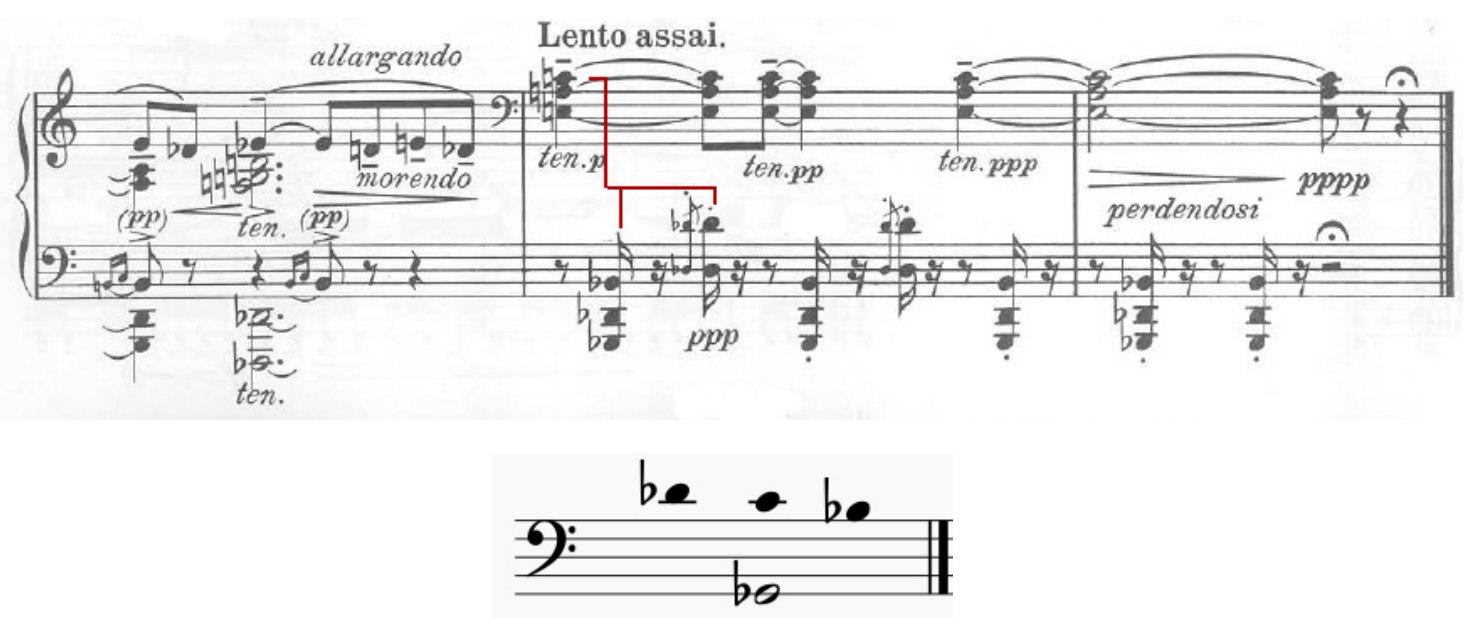

Figure 11 : Karol Szymanowski, Masques, op. 24 (1915-1916),

"Tantris le bouffon» $\left(n^{\circ} 2\right)$, mes. 106-108 et ligne mélodique.

Or, si néanmoins la présence du mi et du la bécarres à la main droite dissone, pourquoi à l'écoute discernons-nous, plutôt qu'un mode de $f a$, davantage la prégnance d'un mode acoustique ? Lors du déroulé du flux sonore, l'oreille réalise ici un phénomène d'assimilation tout à fait intéressant : lorsque l'accord de trois sons survient à la main gauche, les notes do et la de la main droite, bien que leur résonnance se perpétue, sont entendues comme se résolvant par leur jonction sur le si bémol donné à la main gauche. En revanche, le mi bécarre demeure lui bien vivace et prend les traits, par enharmonie, d'un fa bémol, septième mineure. Ainsi, le segment de trois notes dessiné par le timbre des lignes supérieures se voit englobé, par la résonnance du fa bémol, au sein d'une collection rassemblant les caractéristiques du mode acoustique (quarte augmentée, septième mineure) : solb-sib-do-réb-fab.

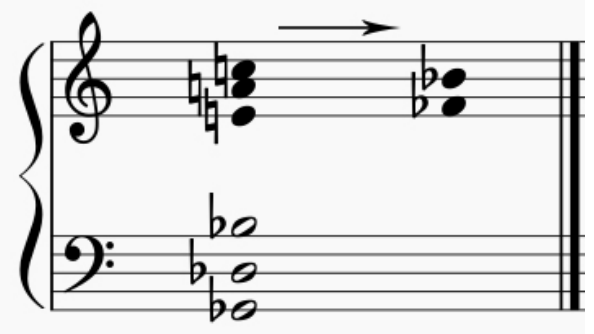

Figure 12 : Lecture harmonique exprimant les caractéristiques du mode acoustique.

Trois mesures avant la fin, la survenance de l'accord de ré bémol dominante altérée marque également une construction remarquable. La main droite et le gruppetto à la ligne basse, considérés isolément, s'attachent, tant par la notation que par leur signifiant harmonico-mélodique, à l'univers de do en présentant sa dominante (sol) sous la forme d'un accord de quatre sons renversé et donnant au mi bémol le rôle d'appoggiature du ré. Par l'adjonction de l'octave grave, la directionnalité de chacune des notes change tandis que l'espace sonore s'envisage autour du pôle fondamental ré bémol. Ne pouvant plus être perçu comme une note tendue, le mi bémol devient 
neutre, et le sol incarne à présent l'altération de la quinte de l'accord. Or, nous constatons que le rapport tritonique de la relation des fondamentales réb-sol créé, d'une façon similaire au rapport solb-do des deux dernières mesures, les conditions de la formation d'un segment de mode de fa qui, par la présence de la septième mineure, s'affirme comme mode acoustique.
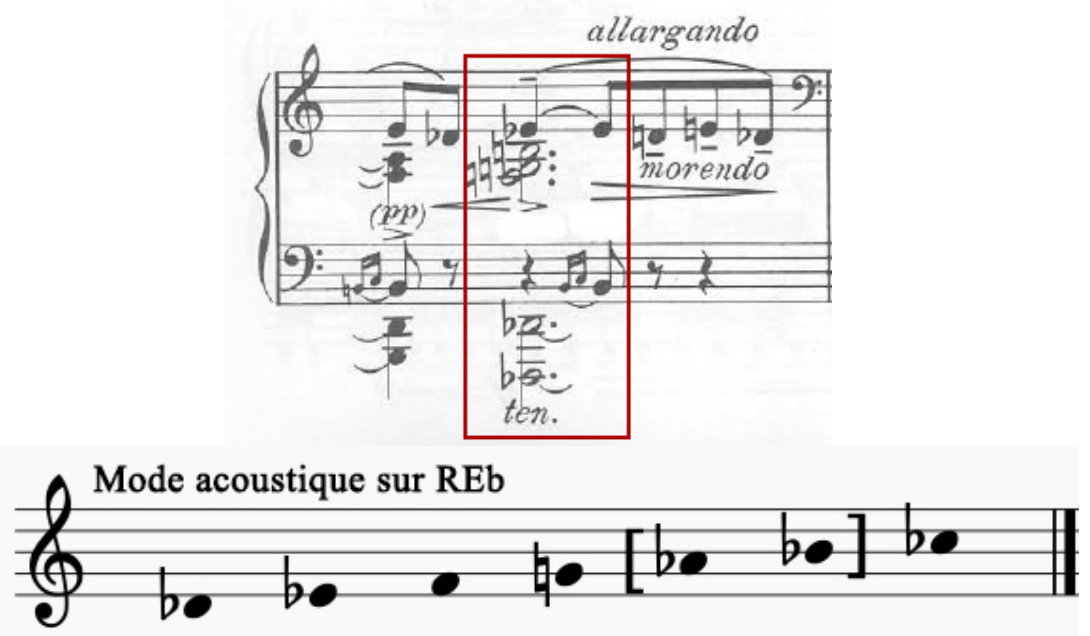

Figure 13 : Karol Szymanowski, Masques, op. 24 (1915-1916), "Tantris le bouffon » (nº 2), mes. 106, accord de neuvième altéré présentant les caractéristiques du mode acoustique.

La réalisation interprétative des quatre dernières croches de la mesure et leur liaison au do de la mesure suivante devrait être déterminée par une conception analytique plus harmonique que mélodique. Nous voyons que Szymanowski, par l'agrandissement progressif de la distance intervallique, présente ici le remplissage chromatique de la tierce do-mi, intervalle agissant comme déterminant modal dans la double potentialité résolutive de l'accord de dominante sur sol.

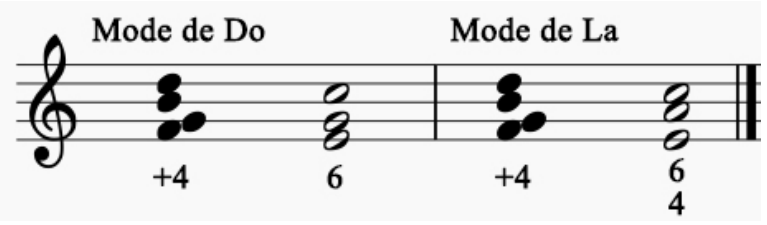

Figure 14 : Double potentialité modale.

Si cette constatation ne peut être d'une importante utilité interprétative et ne se distingue que difficilement à l'oreille, une autre vision est en revanche possible, à l'aune du signifiant harmonique global des deux mesures concernées (mes. 106-107). Le ré bécarre, deuxième croche, incarne la neuvième mineure de l'accord de dominante altéré sur ré bémol par l'abaissement du mi bémol. À ce titre, malgré le soufflet dynamique dégressif, le ré bécarre devrait conserver un poids et un timbre similaire au mi bémol précédent. Le surlignage expressif, nécessaire à la perception de l'augmentation de la tension harmonique induite par l'abaissement de la neuvième, sera effectué par l'allongement léger de la durée de la note. Enfin, les deux dernières croches de la mesure (mi-réb) acquièrent leur sens harmonique a posteriori lorsque surgit à la mesure suivante l'accord de trois sons énoncé à la main gauche. En effet, 
le mi bécarre anticipe auditivement sa présence comme $f a$ bémol entendu au sein de l'accord suivant sur sol bémol. Avec le ré bémol, ils prédéterminent l'implication harmonique de l'accord conclusif, perçu comme septième de dominante, et amplifient le rôle du do comme note de passage entre ré bémol et si bémol (note supérieure de l'accord à la main gauche). Ainsi, l'augmentation de la durée du ré bécarre aura permis de mettre en lumière naturellement la séparation harmonique qui gouverne le groupe de croches. Puis, à travers l'allargando et la diminution dynamique, l'interprète devra néanmoins conduire expressivement les deux dernières croches comme faisant partie intégrante d'un ensemble de quatre notes : mi-réb-do-sib, dont le groupe de tierces mineures appartenant à l'accord sur sol bémol (mi-réb-sib) forme l'ossature.

Les mesures 103-104 méritent également une attention toute particulière car elles déploient, sur l'élément caractéristique de la main gauche fondé sur do, deux cellules à la main droite issues pour l'une de la mesure 3, pour l'autre de la cellule motivique ouvrant le corps de l'œuvre (Allegro moderato. Marcato, mes. 24-25). Or, la coexistence des deux univers voit surgir, par l'association des deux mains, à la mesure 5 avant la fin, une collection de quatre notes dont la succession de tons entiers forme un triton décrivant un segment de mode de fa sur solbémol (do-sib-lab-solb). Puis, l'apparition du sol bécarre sur la dernière croche, à première vue dissonant, permet en fait la jonction avec les deux mesures suivantes par le basculement dans l'univers de ré bémol qui, avec l'altération de sa quinte (sol bécarre), va fournir l'élément essentiel à la formation d'un mode acoustique. L'interprète peut ainsi séparer la mesure en deux parties, la délimitation se situant entre le sol bémol de la main droite, fondement du triton solb-do, et le sol bécarre de la main gauche, départ d'un nouveau triton sol-réb auquel, afin d'affirmer l'assise fondamentale du ré bémol, s'adjoindra d'un la bémol. En retenant un peu la survenance du sol bécarre, la liaison de celui-ci aux notes suivantes de la main droite s'accompagnera naturellement d'une augmentation dynamique ajoutant à la sensation de directionnalité.
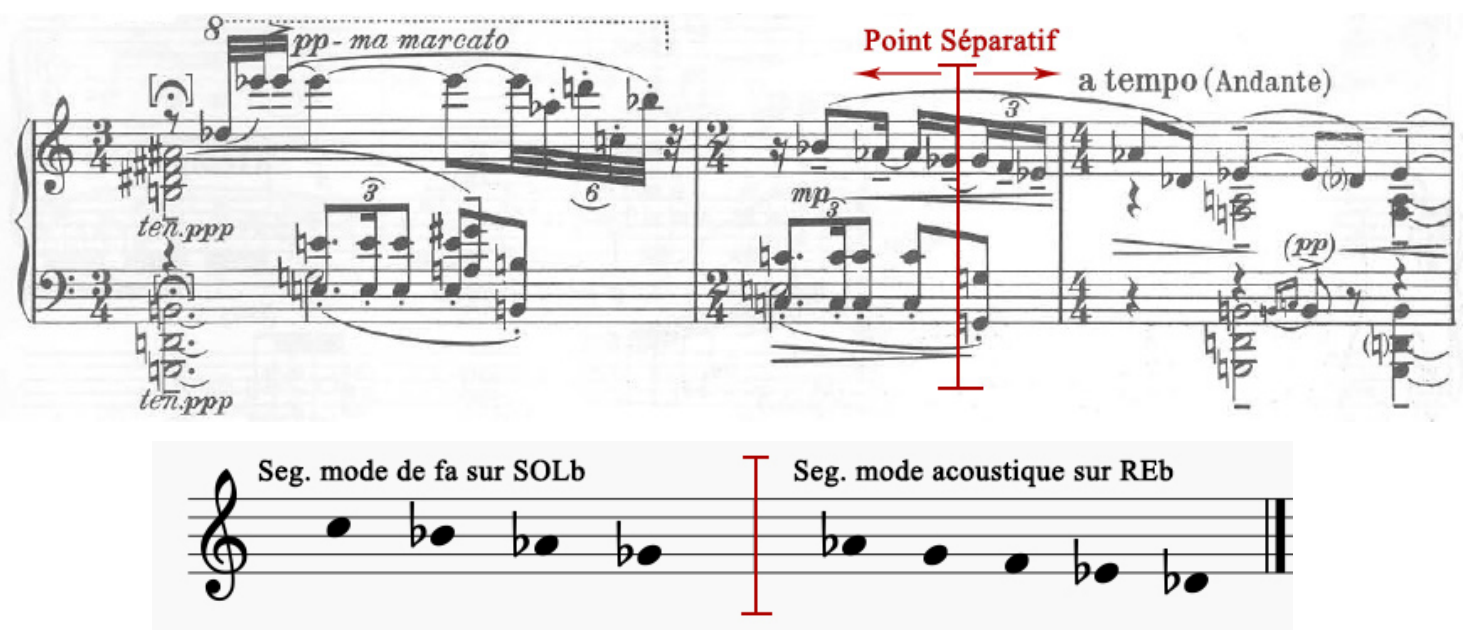

Figure 15 : Karol Szymanowski, Masques, op. 24 (1915-1916), "Tantris le bouffon» (no 2), mes. 103-105, mode de fa sur sol bémol et mode acoustique sur ré bémol. 
La cellule énoncée à la main droite à la mesure 103, présente également, mais de façon brisée, le segment intervallique caractéristique d'un mode de fa sur la bémol (mib-ré-do-sib-lab). La présence du ré bémol initiant la cellule, désignation de l'oscillation quarte juste/quatre augmentée, peut être assimilée à la jonction solb-sol $l^{\natural}$ au sein de l'univers de ré bémol, au milieu de la mesure 104.

Considérant l'intégralité des différents segments intervalliques décrits plus haut, nous nous apercevons que leurs extrémités forment un empilement de trois quintes : solb-réb-lab-mib. Ces quatre notes, en se scindant par l'intermédiaire de la quinte centrale réb-lab, ont la capacité de se présenter comme deux intervalles de neuvième majeure coupés en leur milieu exact, soit : solb-réb-lab et réb-lab-mib.

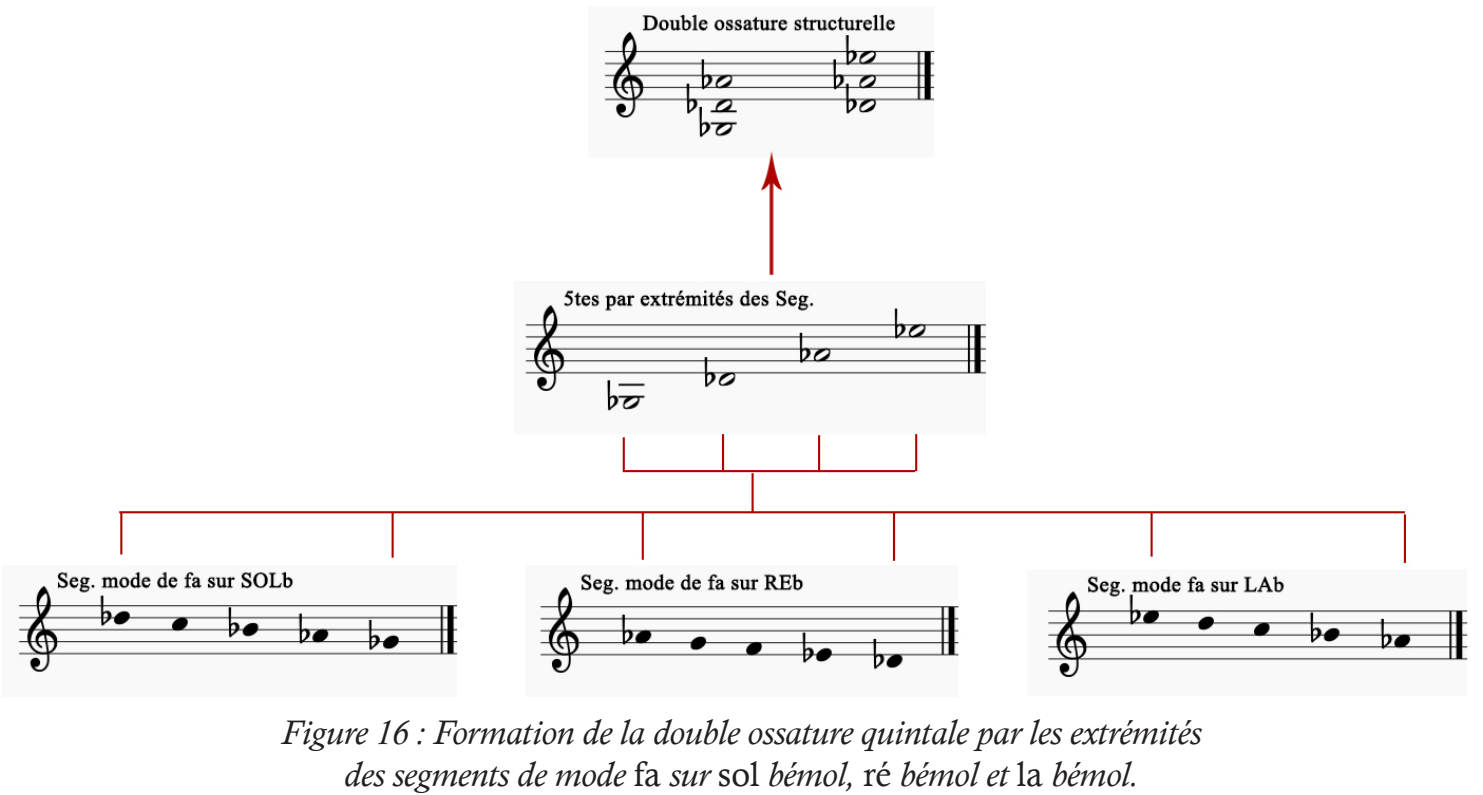

De plus, les altérations des quartes relatives aux caractéristiques intervalliques du mode de $f a$ et du mode acoustique peuvent à leur tour être présentées comme un empilement de deux quintes : do-sol-ré. Cet empilement de quintes do-sol/sol-ré concentre le fondement harmonique de la section "Ancora meno mosso» (mes. 47 et 101), présenté par la main gauche et son motif caractéristique.

Ainsi, les huit dernières mesures (mes. 101-108) qui composent la conclusion de l'œuvre forment un seul groupe uni et cohérent qui trouve sa matrice structurelle au sein de relations intervalliques localisées qui résonnent à l'échelle de la grande forme. Les segments de mode de $f a$ ou de mode acoustique et le geste intervallique qu'ils décrivent par leurs extrémités, formant la double ossature quintale de référence, sont une nouvelle preuve de l'interpénétration constante du microcosme et du macrocosme.

La première page de l'œuvre - dont la conclusion reprend la première cellule motivique saillante exposée à la main droite dès la mesure 3 - témoigne déjà de l'emploi de structures intervalliques propres au mode de $f a$ et au mode acoustique, notamment par les altérations de la quinte des accords. Nous avions pu juger de l'affirmation, à partir de la mesure 3 , du pivot ré bémol par l'adjonction de sa quinte la bémol sur la dernière croche à la main droite. Or, la cellule de la main droite, par 
son déploiement du segment de mode de $f a$, ajoute une strate d'attractivité quintale supplémentaire dont le pôle est la bémol et l'extrémité supérieure mi bémol.

Plus loin, à partir de la mesure 5 , le signifiant harmonique exprimé par la main gauche s'inclinant vers mi bémol, nous retrouvons, avec l'altération de la quinte de l'accord de dominante, les éléments constitutifs d'un mode acoustique : la quarte augmentée et la septième mineure.

Lorsqu'à la mesure 9, le pôle harmonique se déplace vers la bémol, on assiste de nouveau à un jeu sur le double rapport à la fondamentale porté par la structure intervallique du mode de $f a$, aux mesures 10-11 : rapport de quinte mib-lab, et rapport de triton ré-lab. Néanmoins, la présence du ré bémol à la main gauche, bien que perçue comme appoggiature irrésolue du do, trouble l'expérience auditive par sa capacité à produire la potentialité d'une nouvelle appréciation du complexe sonore dans laquelle le balancement mib-rét deviendrait l'oscillation de la neuvième : réb-lab-mib / rét .

Nous souhaitons avancer une théorie pour l'explication des mesures 13-15, point culminant dynamique de l'introduction et particulièrement caractéristique par la cohabitation qu'il propose entre un pentatonisme fondé sur sol bémol à la main droite, et un mode de $f a$ sur do à la main gauche. Outre le fait que nous retrouvons ici le rapport tritonique solb-do qui marquera, comme nous l'avons vu, les ultimes mesures de l'œuvre, nous déterminons que le pentatonisme à la main droite, constitué des notes solb-lab-sib-réb-mib, est naturellement issu du segment de cycle de quintes déployé par les pivots harmoniques des mesures précédentes, hors le sol bémol qui est absent. Or, cette note, qui, en élargissant le cycle des quintes, permet de former à la fois la double ossature structurelle (solb-réb-lab / réb-lab-mib) et propose, avec le si bémol, un geste résolutif qui sera utilisé à la fin de l'œuvre (réb-lab-mib > solb-sib), est justement donnée par enharmonie dans la section "Ancora poco meno » (mes. 20-21). De même, le sol bécarre manquant à la complétion du mode de $f a$ à la main gauche se trouve également comme assise fondamentale dans la section "Poco meno " (mes. 18-19).

Ce passage, qui résume, par la cohabitation de deux espaces, tout à la fois le segment de cycle de quintes structurel et son remplissage intervallique, se situe donc aux mesures 13-14 de l'œuvre, au sein d'un espace introductif s'achevant au début de la section "Allegro moderato. Marcato " (mes. 24), d'une durée totale de 23 mesures. Néanmoins, puisque le trait ornemental (mes. 22) s'organise autour de quatre signifiants harmoniques ayant pour fondamentales, comme nous l'avions fait remarquer, les notes formant deux quintes à distance de demi-ton (réb-lab et ré-la), il serait possible d'envisager une séparation sous-jacente, d'ordre expressif, du groupe de triples croches, liée au déroulement harmonique. Enfin, les quatre notes accentuées, délimitées également et qui achèvent le groupe phraséologique, enchainées ensuite à la mesure suivante, montrent de nouveau, par leur parcours, la coexistence simultanée du pentatonisme (et donc du segment de cycle de quintes ramassé sur lui-même) et de la partie caractéristique du mode de $f a$ (c'est-à-dire, la quarte haussée). 

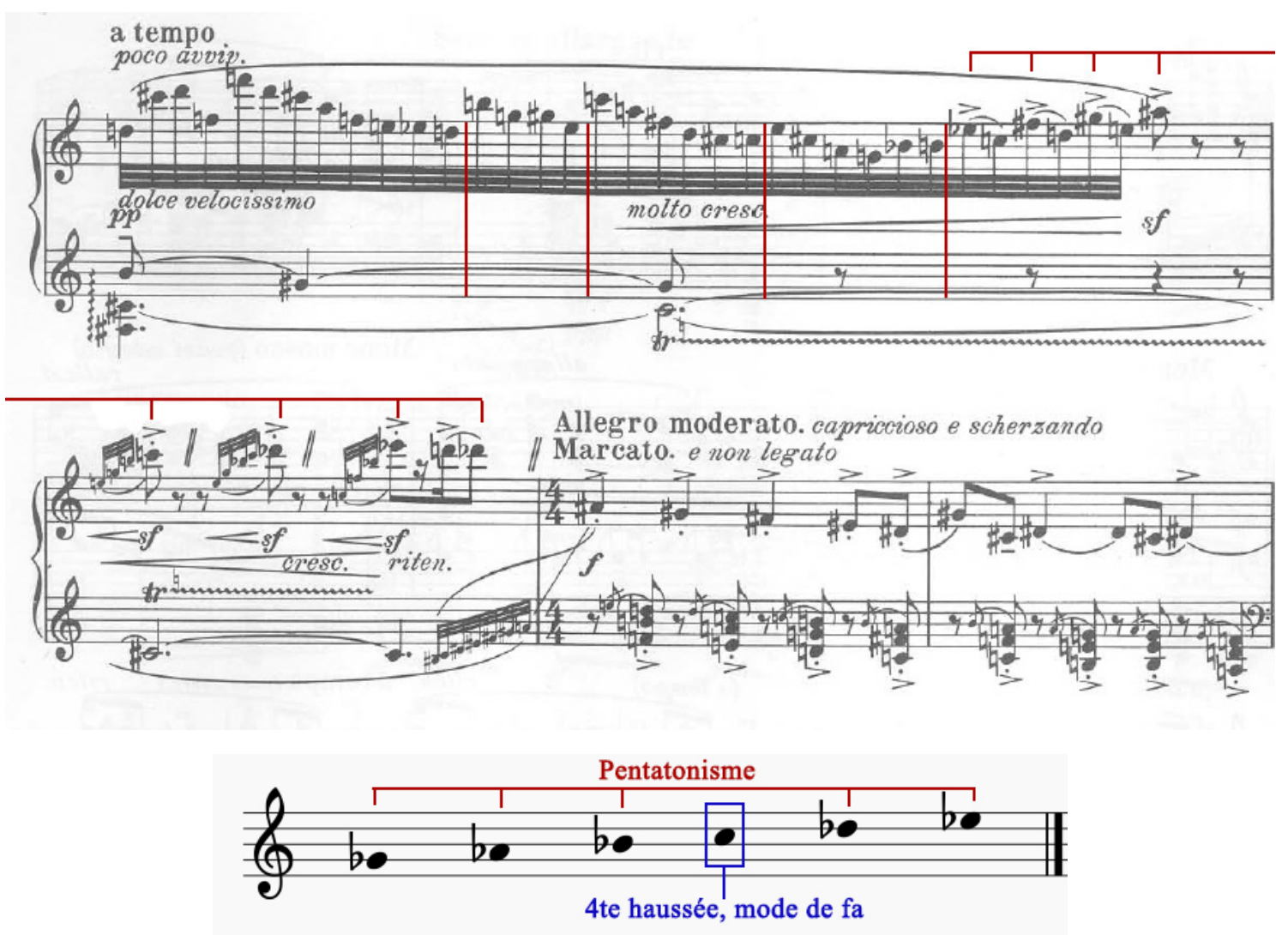

Figure 17: Karol Szymanowski, Masques, op. 24 (1915-1916), "Tantris le bouffon» ( $n^{\circ}$ 2), mesures 22-25, pentatonisme et mode de fa exprimés par les notes accentuées supérieures.

Or, l'adjonction de ces nouvelles barres de mesure au groupe ornemental porte la somme totale de l'introduction à 27 mesures, plaçant de ce fait les mesures 13-14 au centre exact, comme un instant de culmination dynamique qui rassemble les deux constituants à la base de l'organisation du discours.

De quelle façon ces modes d'organisation du discours peuvent-ils trouver leur implication dans la dimension interprétative? Par la mise en œuvre de quels éléments de technique le pianiste pourra, sans exagération musicale, faire ressentir à un auditeur non averti la prégnance de telle ou telle construction intervallique intrinsèque, et comment parviendra-t-il à relier, de façon audible, les différents instants où celles-ci agissent sur l'architecture de l'œuvre, résonnant parfois à plusieurs systèmes de distance ? La connaissance de ce type de dessin structurel, bien réel mais se fondant au sein de complexes sonores qui ne l'expriment pas explicitement, invite davantage, selon nous, à déterminer une trajectoire expressive globale qui procède de la suggestion intellectuelle. La conceptualisation de cette idée mentale issue de l'investigation analytique se manifestera différemment pour chaque interprète, l'un choisissant de ménager des instants de ralentissement du discours afin d'en faciliter l'intelligibilité, l'autre choisissant le surgissement accentuel régulier de certaines notes développant une ossature structurelle, un autre encore agissant sur la métrique et le sentiment de directionnalité. Quoi qu'il en soit, ce type d'organisation de l'espace musical, bien qu'agissant dans une dimension inférieure à la surface d'expressivité tangible, 
détermine ce que l'on pourrait nommer ici le «caractère ». Ce terme n'est pas employé dans sa signification romantique (lié tantôt aux passions, tantôt au raisonnable), mais envisagé comme l'essence qui demeure immuable et qu'il convient de connaître afin de comprendre à fond les différents niveaux architecturels de l'œuvre.

Si du point de vue audible le caractère est de l'ordre de l'éther, il est un instant où celui-ci, pénétrant davantage la surface extérieure de l'espace musical, en détermine plus clairement les contours, bien qu'il soit encore impossible à l'auditeur pris dans le flux sonore d'en percevoir l'implication réelle. En effet, dans le groupe ornemental de triples croches (a tempo, mes. 22), la relation quintale à distance de demi-ton est obtenue par l'identification de quatre fondamentales procédant de l'unification d'un certain nombre de notes mélodiques au sein d'un espace harmonique déterminé, soit trois accords de dominante et un accord parfait dans son second renversement.

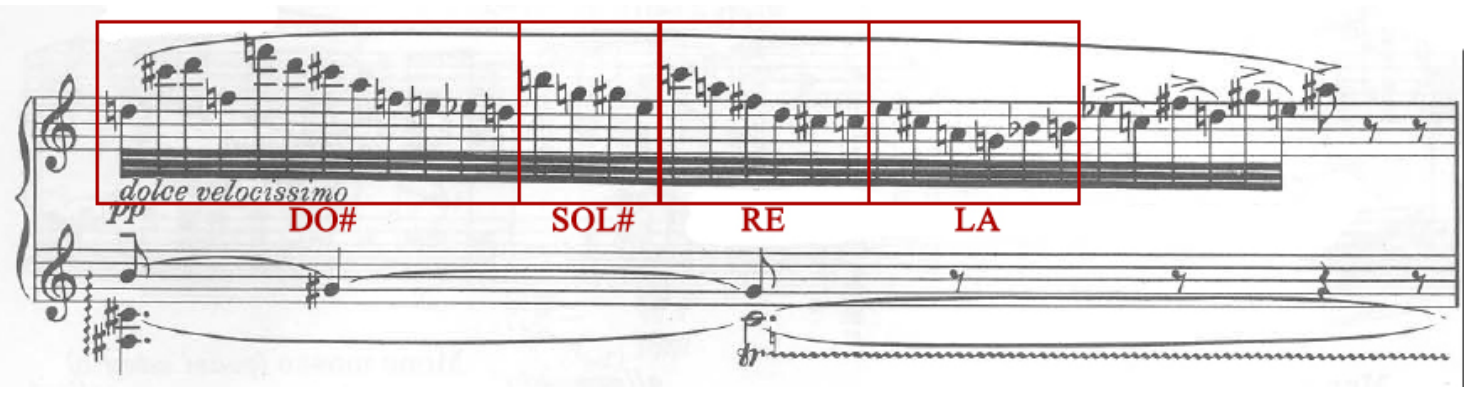

Figure 18: Karol Szymanowski, Masques, op. 24 (1915-1916), "Tantris le bouffon» ( $n^{\circ}$ 2), mes. 22, deux quintes à distance de demi-ton obtenues par l'expression harmonique de l'espace ornemental.

C'est par l'utilisation du rubato que l'interprète sera en mesure de représenter le passage d'un espace harmonique à l'autre ; il s'agit ici d'esquisser plutôt que de souligner. Ainsi, l'articulation légèrement appuyée de l'octave $f a$-fa permettra d'asseoir immédiatement la saveur de dominante par l'adjonction de la sensible de l'accord. Le groupe suivant, second renversement de sol dièse, sera joué en retenant quelque peu afin de laisser à l'oreille un temps suffisant pour apprécier son inscription harmonique malgré sa courte durée. Puis, les deux groupes suivants, manifestant le même dessin interprétatif, se caractériseront d'un rubato dégressif depuis leur première note jusqu'à la quatrième, permettant là encore à l'auditeur de saisir convenablement le dessin des deux septièmes de dominante enchaînées. Enfin, le dernier groupe, séparé des quatre autres et lié, comme nous l'avons vu, à la mesure suivante par la structuration de son dessin mélodique accentué, sera projeté métriquement et dynamiquement dans une progression linéaire dont l'aboutissement se situe sur le mi bémol aigu (ultime mesure de l'introduction).

La relation de demi-ton créée par les fondamentales des quatre groupes cités plus haut, ici difficilement perceptible, s'affirme de façon plus concrète dans l'enchaînement des sections « Poco meno » et «Ancora poco meno » (mes. 18-21). En effet, la superposition quintale à la main gauche, qui singularise ces deux sections, rassemble, dans la quinte supérieure, les quatre fondamentales déployées lors du passage ornemental de triples croches. 

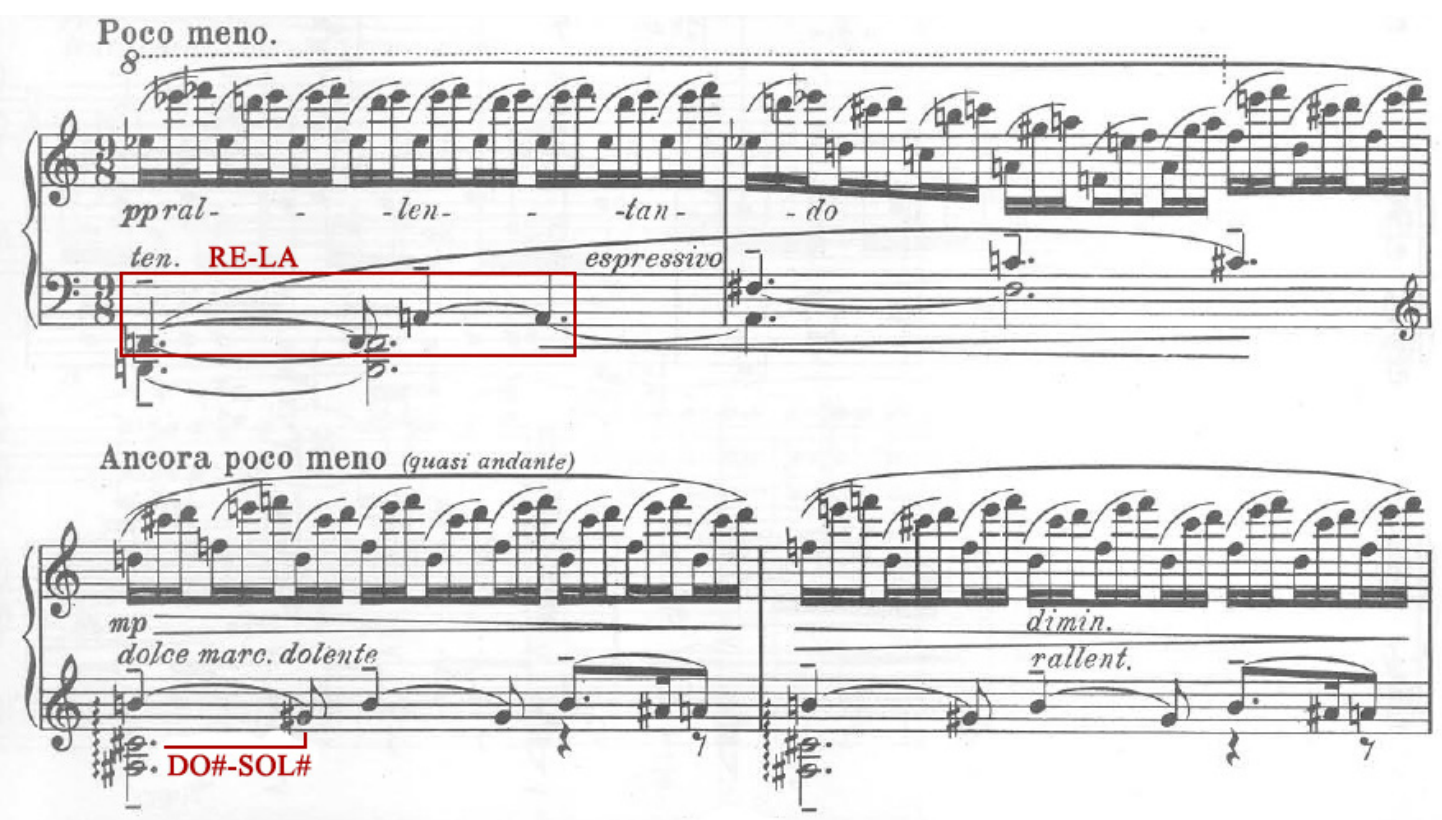

Figure 19: Karol Szymanowski, Masques, op. 24 (1915-1916), "Tantris le bouffon» (n 2), mes. 18-21, deux quintes à distance de demi-ton.

Les deux quintes basses, sol-ré/fa\#-do\#, résonnent de façon tout à fait particulière avec la conclusion de l'œuvre, dans laquelle nous assistons à la basse, à travers le mouvement tritonique du passage d'une dominante à l'autre ( $s o l v>$ réb v), à l'incarnation claire de la quinte sol-ré, puis lors de la résolution finale, à la présentation de la quinte solb-réb. Si néanmoins la section "Ancora poco meno " (mes. 20-21) présente une superposition quintale qui, à l'aune du mouvement cadentiel achevant l'œuvre, peut à première vue s'interpréter comme un rapport de subordination V-I, il s'agit cependant, afin de clarifier le statut de la quinte ré-la vis-à-vis de sol-ré, d'adopter une vue la plus complète possible. La somme des différents instants au cours de l'œuvre au sein desquels survient une telle cohabitation quintale nous permet d'obtenir deux nouvelles quintes supplémentaires qui se placent naturellement dans la continuité des deux cycles esquissés aux mesures 18-21 : la quinte do-sol, issue de la section "Ancora meno » (mes. 47 et 101), et la quinte lab-mib issue de la section «Vivace» (mes. 59).

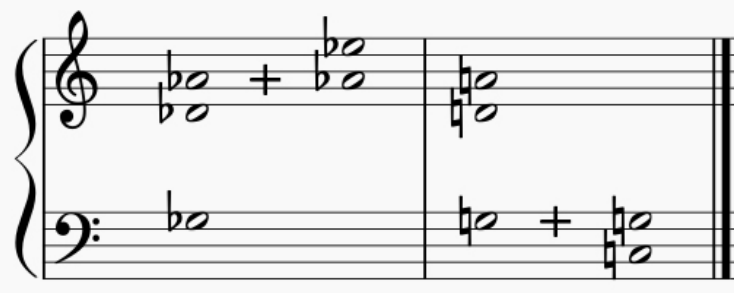

Figure 20: Deux cycles de quinte à distance de triton.

Les deux nouveaux cycles obtenus, à distance de triton, ont, comme nous le savons, la capacité de présenter la double ossature structurelle (solb-réb-lab + réb-lab-mib) 
ainsi que la double altération de leurs quintes (do-ré et sol-la). Toutefois, l'adjonction de la quinte do-sol au cycle sol-ré-la vient réévaluer la fonction de ré-la comme participative d'une ossature de neuvième. À ce titre, les quatre dernières mesures de l'œuvre montrent l'implication harmonique de cette ossature, sous les traits d'une neuvième de dominante, et sa résolution (bien que pluridimensionnelle) vers le pôle do.

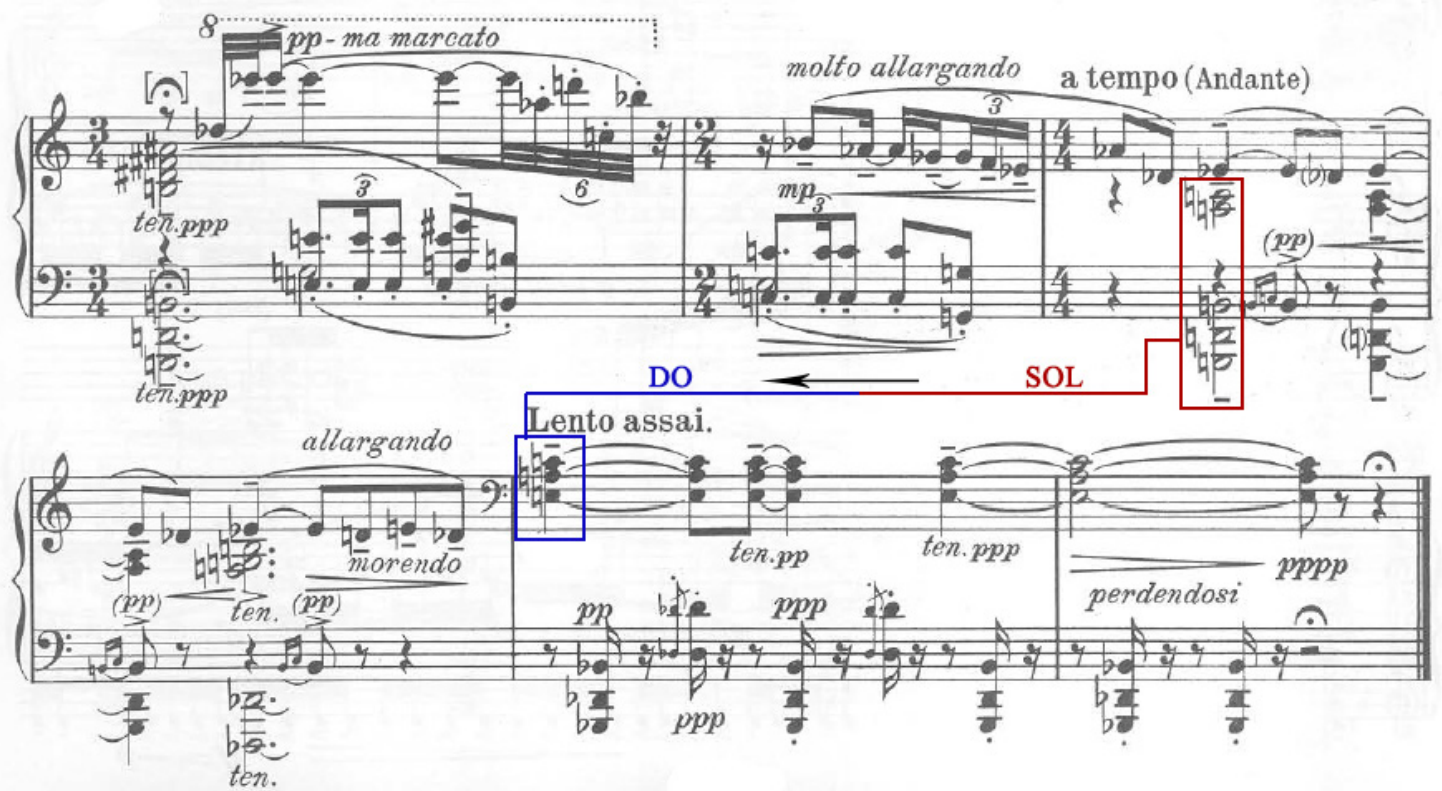

Figure 21 : Karol Szymanowski, Masques, op. 24 (1915-1916), "Tantris le bouffon» (n 2), mes. 103-108, forme d'aboutissement de l'accord de neuvième sur sol.

Afin de percevoir la réalité sonore de la superposition quintale et sa détermination d'une cohabitation d'un accord de dominante sur une pédale de tonique, l'interprète devra, à la section " Poco meno " (mes. 18-19), appuyer à la main gauche le sol davantage que le ré. De cette façon, l'assise tonique aura la capacité de résonner toute la mesure (mes. 18), tandis que le ré, par le déploiement progressif de l'accord de septième de dominante, acquerra naturellement sa dimension de fondamentale, et bien que relâché à la deuxième mesure de la section (mes. 19), il persistera néanmoins dans la mémoire de l'auditeur. Dans la section "Ancora poco meno» (mes. 20-21), le pianiste prendra garde de ne pas serrer l'énoncé de l'arpège à la main gauche mais, au contraire, marquera une très légère césure entre le $f a$ dièse, assise tonique, et $d o \#$-si caractérisant l'accord de dominante afin que l'auditeur puisse ressentir que ce sont là deux éléments distincts qui coexistent dans un équilibre précaire et non pas un seul accord de $f a$ dièse appogiaturé. La main droite, dans les deux sections, dessinera chaque groupe de triolets par un mouvement circulaire du poignet, dans le sens inverse des aiguilles d'une montre. 


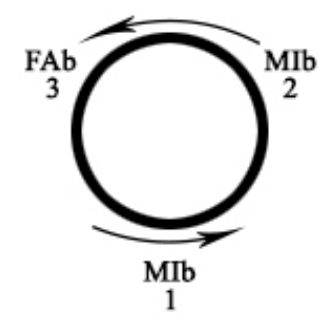

Figure 22 : Geste de rotation du poignet.

L'abaissement du poignet sur le pouce permet ainsi l'adjonction d'un poids supplémentaire qui détermine l'articulation du phrasé et la proéminence de la trajectoire mélodique façonnée par les premières notes des triolets.

Dans ces deux sections, la superposition quintale s'articule en présentant dans la partie inférieure une note fondamentale à laquelle s'adjoint sa quinte qui, renforçant d'abord son assise, génère ensuite au-dessus d'elle un agrégat qui, par sa réalisation, définit tout à la fois la note grave comme tonique par rapport à lui, et se définit lui-même comme accord de dominante. Or, dans la section "Ancora meno » (mes. 47 et 101), le principe s'inverse : la triade de sol majeur, premièrement énoncée à la main gauche, donne suite à une cellule motivique dont la directionnalité mélodique place le do comme aboutissement, et dont l'empreinte harmonique, ôtée des notes de passage, désigne un accord de do majeur.

À ce titre, l'interprète devra révéler l'attractivité entre les deux pôles qui se manifeste à cet instant en surmontant la difficulté inhérente à la physionomie du complexe sonore, c'est-à-dire le passage d'un élément vertical dont la perception est immédiate, à un substrat horizontal dont l'essence expressive est étalée dans le temps. Pour ce faire, il doit en premier lieu établir la trajectoire interprétative de la cellule motivique : la séparation en deux groupes phraséologiques ne doit pas nuire à la sensation d'unification harmonique qui doit ici prévaloir. Le si devrait donc s'incliner vers le $d o$, sans qu'il ne s'entende de coupure entre ces deux notes; en revanche, la délimitation phraséologique s'incarnera par un appui plus important et, corrélativement, une élongation de la durée sur la note do, affirmant de ce fait son importance structurelle vis-à-vis des autres notes de la triade ( $m i$ et $s o l$ ). En second lieu, l'accord de sol majeur verra sa note supérieure (si) se détacher des autres par l'usage d'un timbre plus saillant. Cette sonorité particulière est réalisée en relevant le pouce à l'oblique de la touche, afin que l'enfoncement ne se fasse pas, comme à l'accoutumée, par le plat du pouce, mais par son extrémité, voire, selon l'instrument et sa sonorité, par le coin de l'ongle. La mise en exergue du si va naturellement conduire à la perception du déplacement mélodico-harmonique qui s'effectue par le passage de l'agrégat à la cellule motivique et vice-versa ; le si étant entendu comme allant au do, puis le do revenant au si à la fin de la deuxième mesure de la section.

Ce choix interprétatif permet aux deux univers, sur sol et $d o$, de coexister à force égale, sans qu'il soit possible de déterminer si nous sommes en présence d'une relation $\mathrm{v}(s o l)-\mathrm{I}(d o)$, ou bien $\operatorname{IV}(d o)-\mathrm{I}(s o l)$. L'équilibre qu'affichent ces deux pôles est révélé par la présentation en cycle des quintes des fondamentales sur lesquelles se fondent les espaces harmoniques de l'œuvre, en disposant au centre la quinte enharmonique où 
s'effectue le basculement, et montrant, de ce fait, aux deux extrémités du cycle, les notes sol et do.

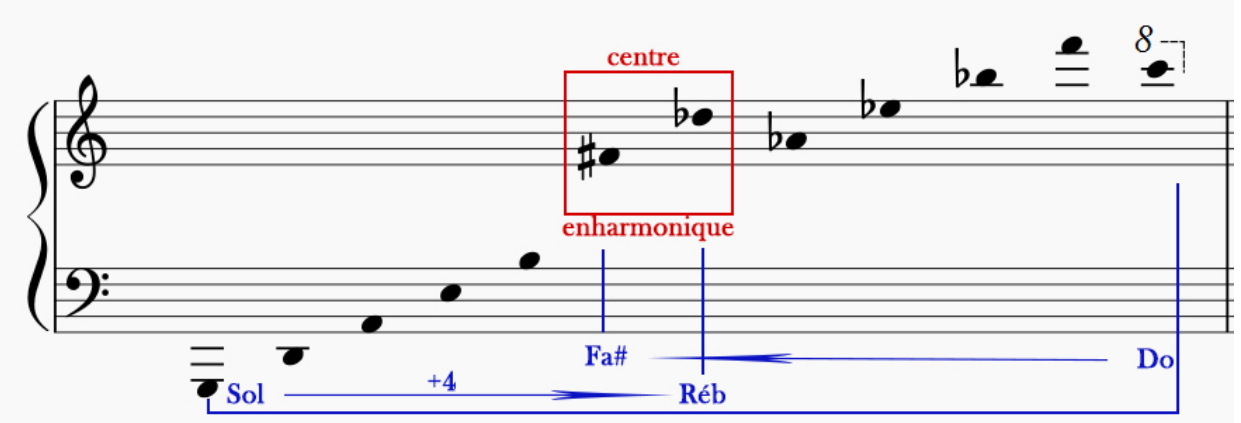

Figure 23 : Fondamentales issues du relevé topographique des espaces harmoniques des sections de "Tantris le bouffon" de Karol Szymanowski (Masques, op. 24, 1915-1916).

Le basculement enharmonique est placé au centre et met en lumière le rapport extrême sol-do.

La section centrale "Vivace» (mes. 54-63), acquiert, au titre du cycle des quintes, une dimension particulièrement riche de potentialité structurante sur laquelle il est nécessaire d'insister. Dans les deux mesures qui précèdent la section, nous assistons à la cohabitation de deux fondamentales, respectivement si bémol à la main droite, qui s'articule, par la présence du cycle de tierces mineures, autour d'un segment d'accord de neuvième de dominante sans sensible, et $f a$ à la main gauche, générant un accord de septième de dominante altéré. Lorsque la section "Vivace » est initiée, l'univers de si bémol se perpétue encore dans les petites notes de la main droite, tandis que le reste du complexe bascule vers si bécarre ${ }^{12}$, présentant, si l'on replace la somme des notes concernées en classe de hauteur, une gamme dont trois notes sont mouvantes, la tierce, la septième et la sixte, oscillant ainsi entre le mode majeur et les modes mineurs mélodiques et harmoniques, et investis également de la capacité de former un accord de septième de dominante par le haussement de la tierce.

12 Notons à ce titre les $s f$ qui soulignent, par l'accentuel, l'harmonisation de si bécarre sous les traits d'une septième de dominante comme point focal. 


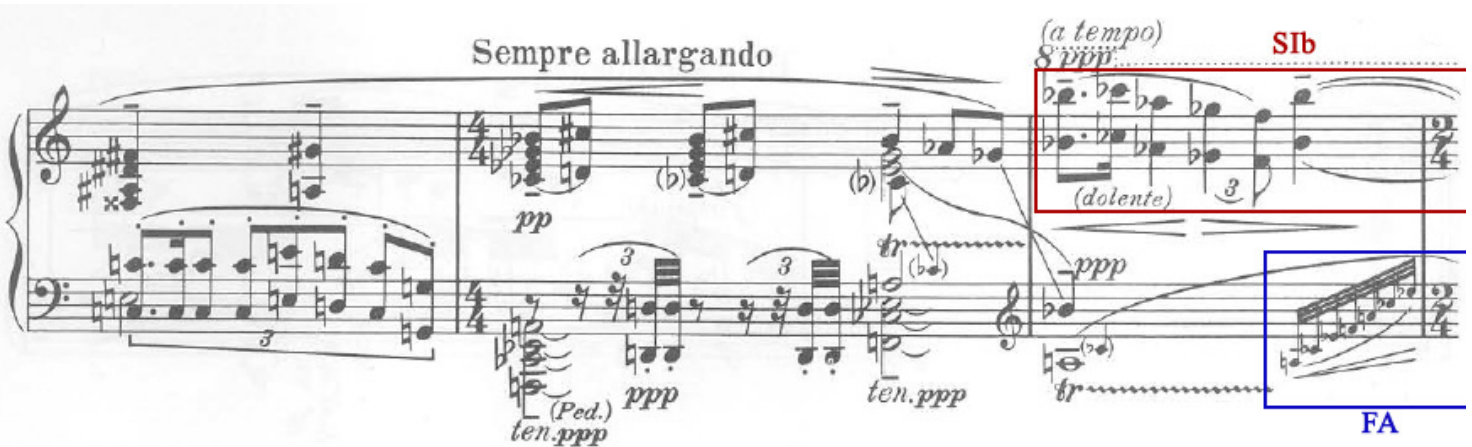

Allargando. Vivace. (non troppo)

$\mathrm{SIb}$

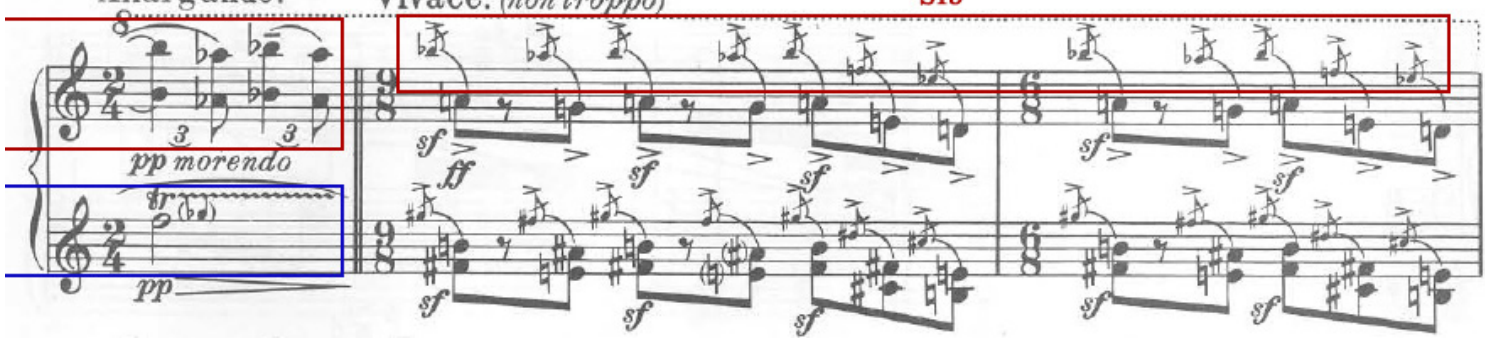

Figure 24 : Karol Szymanowski, Masques, op. 24 (1915-1916), "Tantris le bouffon " (n 2), mes. 50-55, cohabitation des univers harmoniques de si bémol et fa.

La centralisation des deux premières mesures de la section (mes. 54-55) autour de l'univers de si bécarre est remarquable pour deux raisons. La première est que l'accord de dominante altéré sur fa, déployé clairement à la fin du premier système, a la capacité, par enharmonie et via la relation de triton, de former un accord de dominante sur si bécarre ; le haussement du $f a$ au $f a$ dièse, pour obtenir la quinte juste de l'accord, semble, par anticipation, déjà esquissé par le trille fa-solb de la main gauche. La seconde est que les accords de dominante, au nombre de trois, formés par la rencontre des deux mains, se fondent sur les fondamentales $s i$, fa dièse et mi. Or, si nous disposons ces trois notes sous la forme d'un segment de cycle de quintes, mi-si-fa \#, nous obtenons le rapport IV-I-V, dont nous avons précédemment montré les différentes capacités d'incarnation au sein de l'espace musical. Szymanowski met ici en œuvre le même procédé d'organisation harmonique utilisé dans la section "Ancora meno " (mes. 47) : les fondamentales si et mi, aux extrémités des deux premières mesures de la section "Vivace » (mes. 54-55), figurent, à l'échelle architectonique la plus large, les septièmes des accords structurels sur ré bémol et sol bémol ; elles sont prises ainsi dans le dessin du geste global V-I (réb-solb), tel que présenté dans l'espace cadentiel terminal de l'œuvre. Néanmoins, le retour sur si bécarre, au début de la deuxième mesure de la section, fait apparaître l'enchaînement mi-si comme attaché à une relation plagale IV-I, le si bécarre acquérant alors sa véritable dimension de point central. 


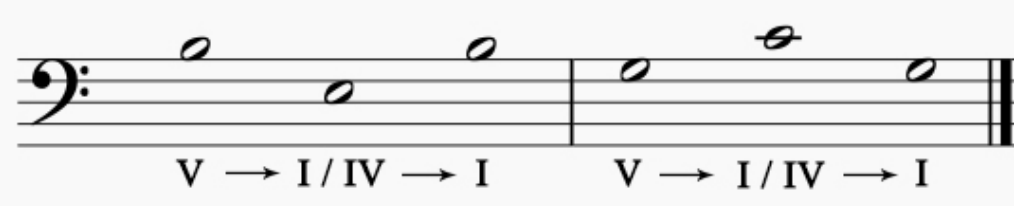

Figure 25 : Relations de degrés aux mesures $54-55$ ("Vivace "), similaires aux mesures 47-48 ("Ancora meno »).

À partir de la troisième mesure de la section (mes. 56), et jusqu'à la fin du système, la main droite déploie les constituants du mode pentatonique basé sur sol bémol, déterminé par les notes des deux ossatures structurantes de l'œuvre. Là encore, Szymanowski parvient à créer une relation de symétrie à l'échelle du système par l'adjonction du fa bémol au mode pentatonique (à la troisième mesure, par la petite note de la main droite), et du do bécarre (à la première mesure, par la petite note de la main gauche). L'intégration de ces notes au pentatonisme forme un mode acoustique sur sol bémol qui résonne, comme nous l'avons vu, avec la structuration intervallique sous-jacente des espaces introductif et conclusif de l'œuvre.

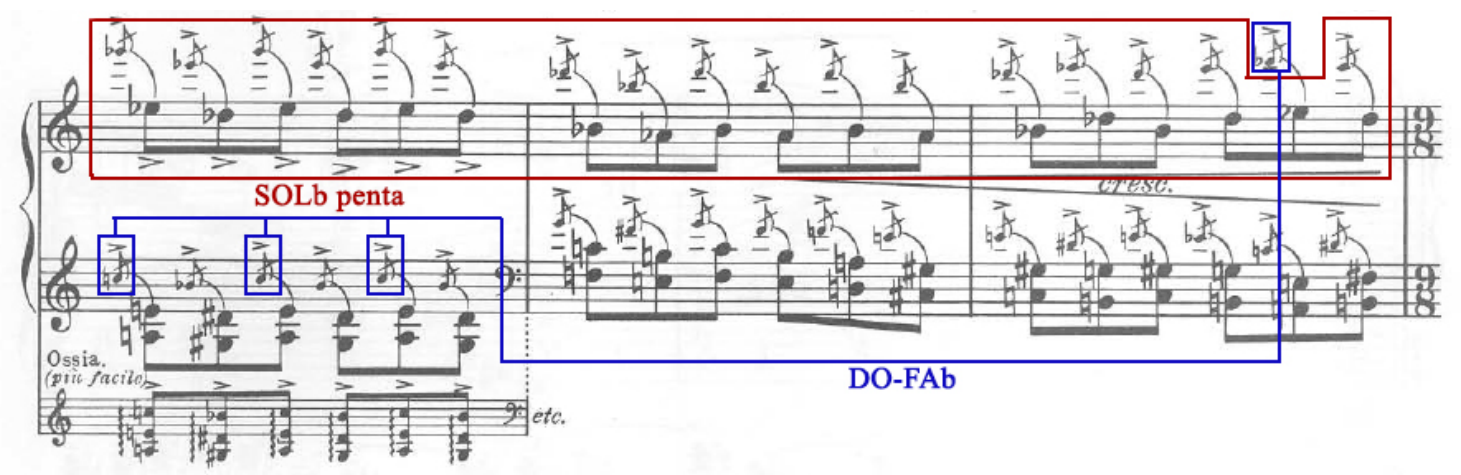

Figure 26: Karol Szymanowski, Masques, op. 24 (1915-1916), "Tantris le bouffon» (n’ 2), mes. 56-58, mode acoustique virtuel sur sol bémol par l'adjonction du do et du fa bémol au pentatonisme sur sol bémol.

Dans ce même système, les quintes à distance demi-ton présentées à la main gauche aux mesures 56 et 57 méritent également une attention toute particulière. Trois groupes de deux quintes à distance d'un demi-ton sont donnés avec un nombre de réitérations retranché de deux croches à chaque nouveau groupe (6-4-2). Or, certaines de ces quintes forment, avec la petite note qui les précède, des triades mineures - la-(do)-mi, ré-(fa)-la, do\#-(mi)-sol\# - et une triade majeure si l'on inclut la mesure 58 avec $s i-(r e ́ \#)-f a \#$. 


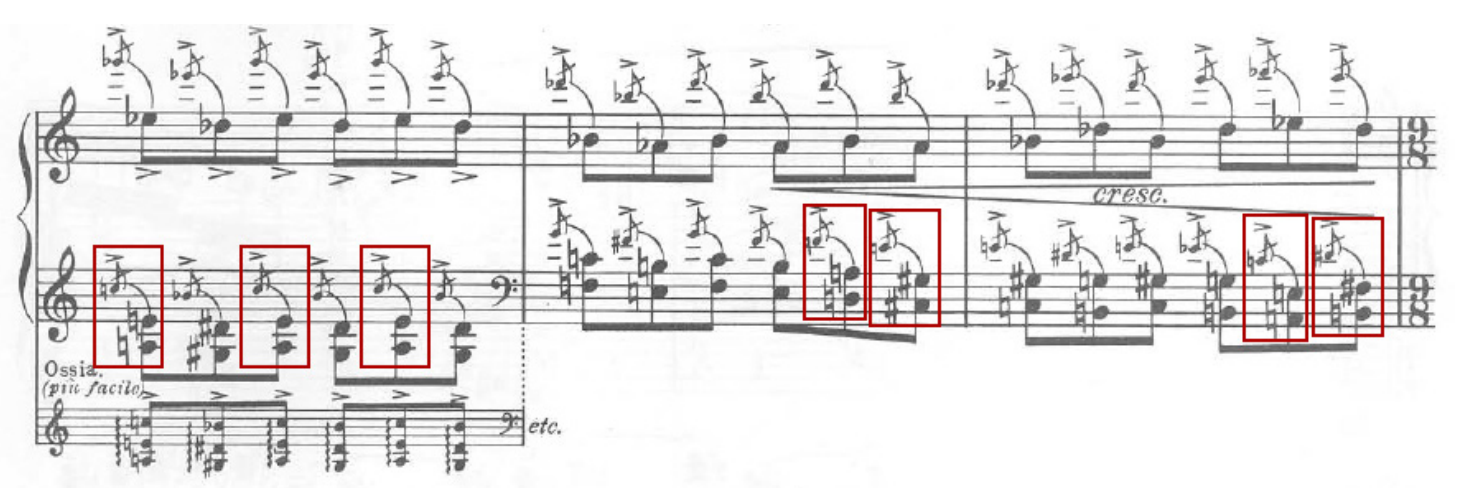

Figure 27 : Karol Szymanowski, Masques, op. 24 (1915-1916),

"Tantris le bouffon $"\left(n^{\circ} 2\right)$, mes. 56-58, quatre triades.

Ces quatre triades ont la capacité de générer un cycle de quintes dont seules les notes sol et la dièse sont absentes. Notons toutefois que ces deux notes forment les extrémités du complexe sonore de la section «Ancora meno » (mes. 47 et 101).
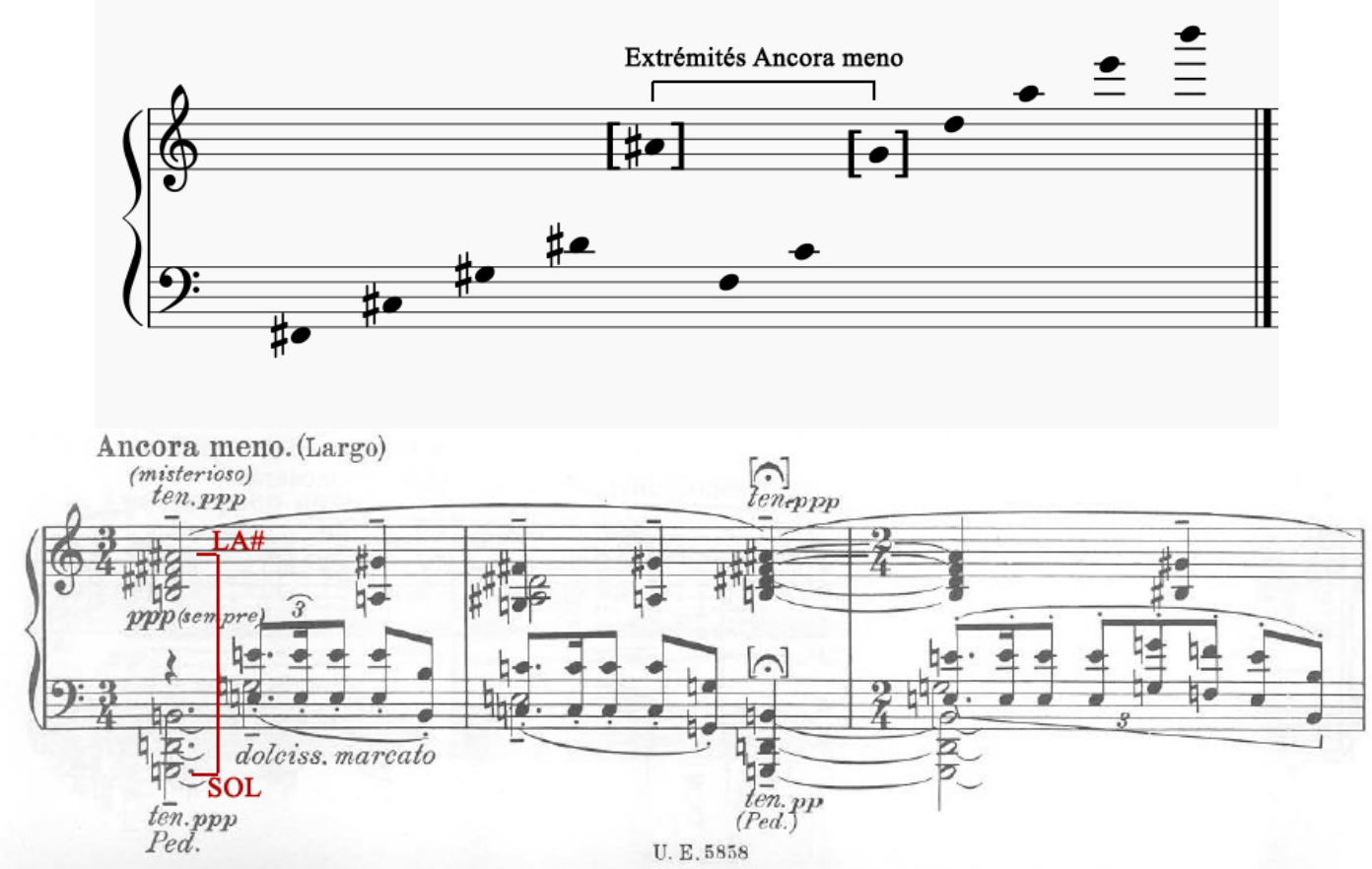

Figure 28: Karol Szymanowski, Masques, op. 24 (1915-1916), "Tantris le bouffon » (n 2), mes. 47-49, les notes sol et la dièse figurent aux extrémités de l'espace musical.

Néanmoins, cette vision exclut les relations de demi-tons dans les trois groupes de quintes, notamment les groupes 1 et 3 qui présentent le rapport do\#-sol\# > ré-la que nous retrouvons dans les sections «Poco meno » et «Ancora Poco meno" (mes. 18-21), et le rapport sol\#-ré\# > la-mi que nous rencontrons par enharmonie au quatrième système de cette page (mes. 59). Regroupées en segment de quintes, c'est-à-dire do\#-sol\#-ré\# et ré-la-mi, nous pouvons distinguer les trois quintes, à distance de demi-tons, qui sont absentes pour la complétion du cycle, soit : sib-fa, si-fa\# et do-sol. Si la quinte do-sol imprime sa marque dans la section significative "Ancora meno" (mes. 47 et 101), le rapport sib-fa/si-fa\# s'incarne dans la section centrale «Vivace» (si-fa\#; mes. 54-55), 
et les deux mesures précédentes (sib-fa ; mes. 52-53) ; mais également par l'assise quintale des mesures 86-88 (si-fa\#) et la transition au "Largo " (sib-fa; mes. 91).

Lorsque l'on ajoute au segment de cycle les deux quintes centrales laissées de côté $(f a-d o / m i-s i)$ qui s'incarnent également dans les deux dernières mesures de la page (mes. 61-62), seulement trois notes demeurent absentes : la\#-sol-fa\#. Or, nous constatons que nous retrouvons ici deux notes communes ( $l a \#$-sol) aux notes absentes du cycle précédemment obtenu par la combinaison des quatre triades, auxquelles s'adjoint le $f a$ dièse. L'intervalle de tierce formé par fa\#-la\# est, par enharmonie, la base de l'identité expressive de l'accord structurel de sol bémol majeur, triade ou par extension septième ou neuvième de dominante. Cet intervalle est dessiné dans la section "Ancora meno » (mes. 47 et 101), à la main droite dans la ligne supérieure, le sol dièse étant alors perçu comme une note de passage. Toutefois, le sol bécarre, à l'extrémité grave du complexe sonore, donne un nouveau remplissage de la tierce et crée de ce fait la collection la $\sharp$-sol-fa $\#$.

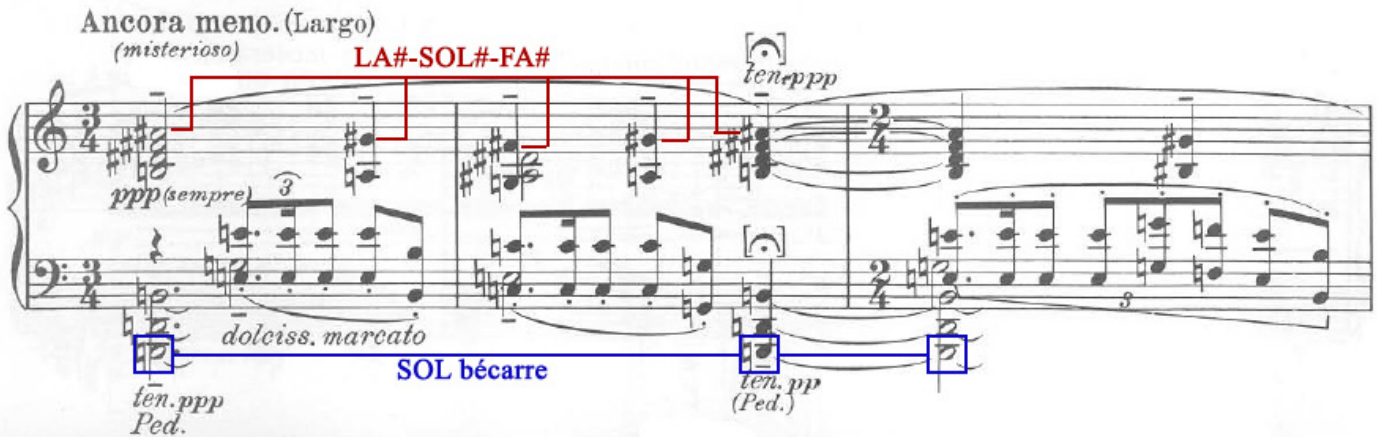

Figure 29: Karol Szymanowski, Masques, op. 24 (1915-1916),

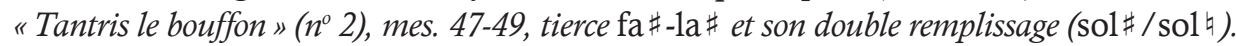

La disposition intervallique caractéristique de cette collection nous amène à la rapprocher des trois dernières notes de l'œuvre à la main droite : mi-réb-do.

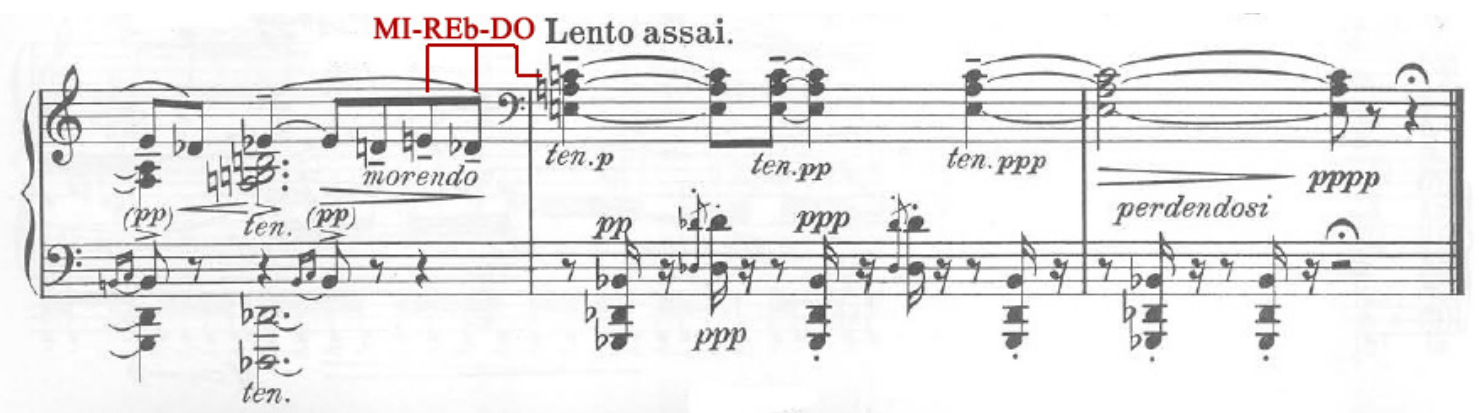

Figure 30 : Karol Szymanowski, Masques, op. 24 (1915-1916),

"Tantris le bouffon" ( $\left.n^{\circ} 2\right)$, mes. 106-108, tierce do-mi et son remplissage ré bémol.

Par l'interpénétration des deux collections, nous pouvons disposer ces six notes sous la forme de deux triades à distance de triton, fa\#-la\#-réb et do-mi-sol, qui nous permettent de procéder à plusieurs observations. En premier lieu, nous constatons la manifestation du point tournant enharmonique $f a \#$-réb au sein de la première triade, 
figurant la capacité que possèdent ces deux notes à revêtir tel ou tel masque altératif. En second lieu, considérant la section "Ancora meno » (mes. 47 et 101), et les deux dernières mesures de l'œuvre, nous observons que la double triade ne parvient jamais à s'incarner de façon complète : le ré bémol est absent dans la section "Ancora meno ", tandis qu'à la fin de l'œuvre (mes.107), le sol, par un procédé d'évitement similaire à celui d'une cadence rompue, est remplacé par un la. Cette relation entre les deux triades semble être déterminante dans l'élaboration à l'instant caractéristique de cohabitation tritonique main gauche/main droite que nous trouvons aux mesures 13-15, dont le $f a$ dièse, bien qu'encore attaché à l'univers du mode de $f a$ qui prédomine dans l'introduction, sera haussé au sol lors de la réitération du passage inversé aux mesures 82-85. De même, aux mesures 104-105, la greffe, dans la section "Ancora meno", de la cellule motivique issue de la section "Allegro moderato » (mes. 24), est là encore révélatrice de cette relation triadique et nous fait envisager le complexe sonore relatif des mesures 24-25 et 69 à l'aune de ce rapport.

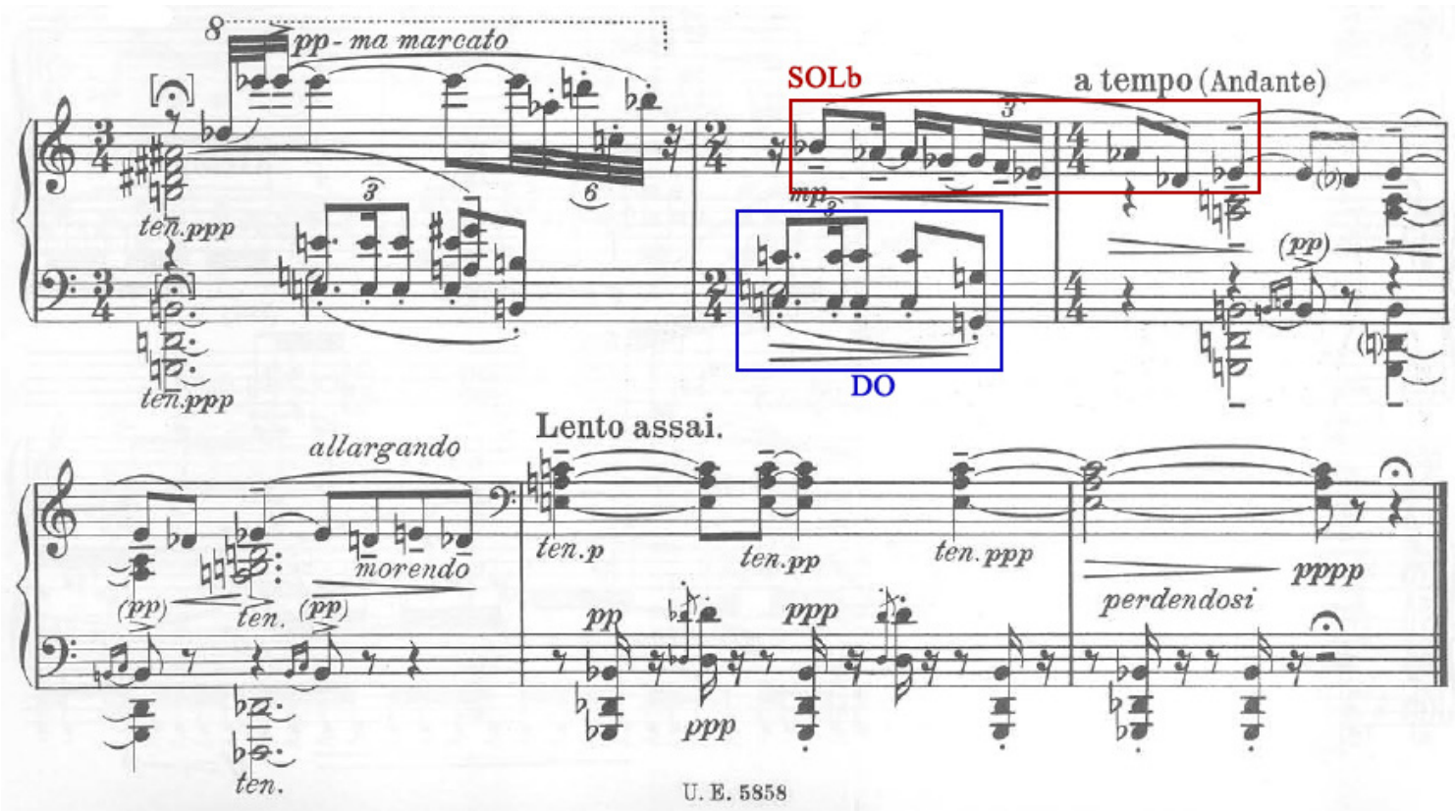

Figure 31 : Karol Szymanowski, Masques, op. 24 (1915-1916), "Tantris le bouffon" ( $n^{\circ}$ 2), mes. 103-108, relation tritonique issue de la double triade.

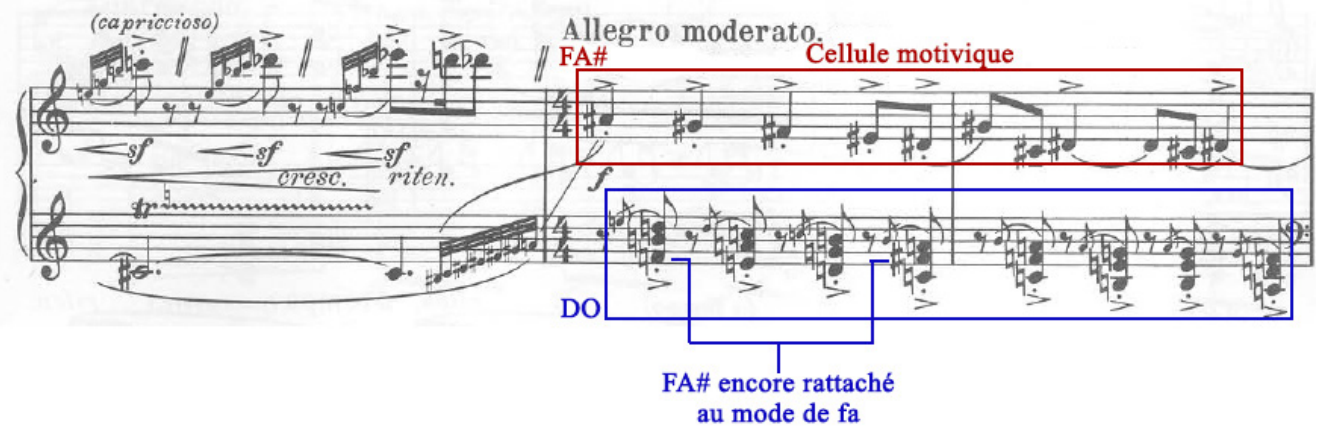

Figure 32 : Karol Szymanowski, Masques, op. 24 (1915-1916),

"Tantris le bouffon" $\left(n^{\circ} 2\right)$, mes. 23-24, relation tritonique issue de la double triade. 


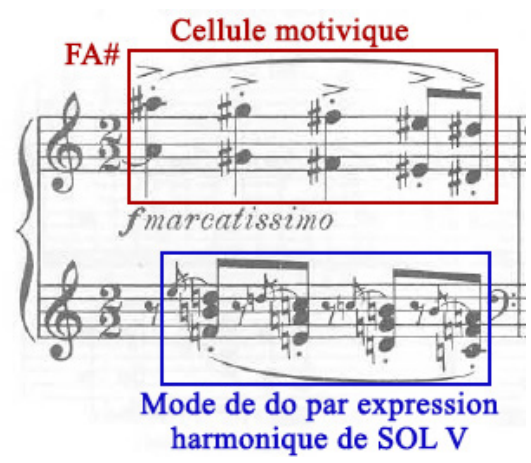

Figure 33 : Karol Szymanowski, Masques, op. 24 (1915-1916),

"Tantris le bouffon" ( $\left.n^{\circ} 2\right)$, mes. 69, relation tritonique issue de la double triade.

À partir de cette démonstration analytique qui révèle une nouvelle part de la construction intrinsèque de l'œuvre, l'interprète est à même d'élaborer une trajectoire interprétative déterminée, lorsque cela est possible, par la mise en exergue des éléments d'organisation du discours. Ainsi, aux mesures 54-55, au-delà de l'accentuel demandé par le $s f$ sur l'accord de dominante fondé sur $s i$, le dernier triolet de chaque mesure devrait être investi d'un appui plus conséquent afin de marquer l'implication des accords de $f a$ dièse et $m i$. Chaque groupe de notes sera articulé par un geste similaire ; l'intensité sonore résultera de la hauteur et du poids de chute de l'avant-bras : le cinquième doigt doit tomber sur la petite note tandis que la main est entraînée dans un mouvement de rotation du poignet vers la gauche, projetant le pouce à l'intérieur du clavier dont l'élan détermine la valeur de son accentuation. Aux mesures 56-57, la succession des trois groupes de quintes à distance de demi-ton devrait montrer pour chacun une délimitation de l'ordre d'un léger dessin phraséologique, marqué d'une césure avant le début de chaque groupe. Dans une logique expressive similaire, l'usage d'un rubato sur les deux premières quintes des groupes s'avèrera utile, par le ralentissement métrique et l'appui qu'il produit, pour accroître la compréhension auditive de l'importance que revêtent ces relations quintales de demi-ton, eut égard de la structuration de l'œuvre. Enfin, le soufflet expansif qui s'affirme dans la dernière mesure du système, doit amener l'interprète à déterminer la valeur dynamique qui le précède. Tandis que les deux mesures initiales du "Vivace » (mes. 54) sont données dans un ff, l'emploi d'une nuance plus faible à partir du second système de la section (mes. 56) viendra en corrélation avec le rubato de façon tout à fait naturelle et permettra de focaliser d'autant l'attention de l'auditeur sur l'instant. 


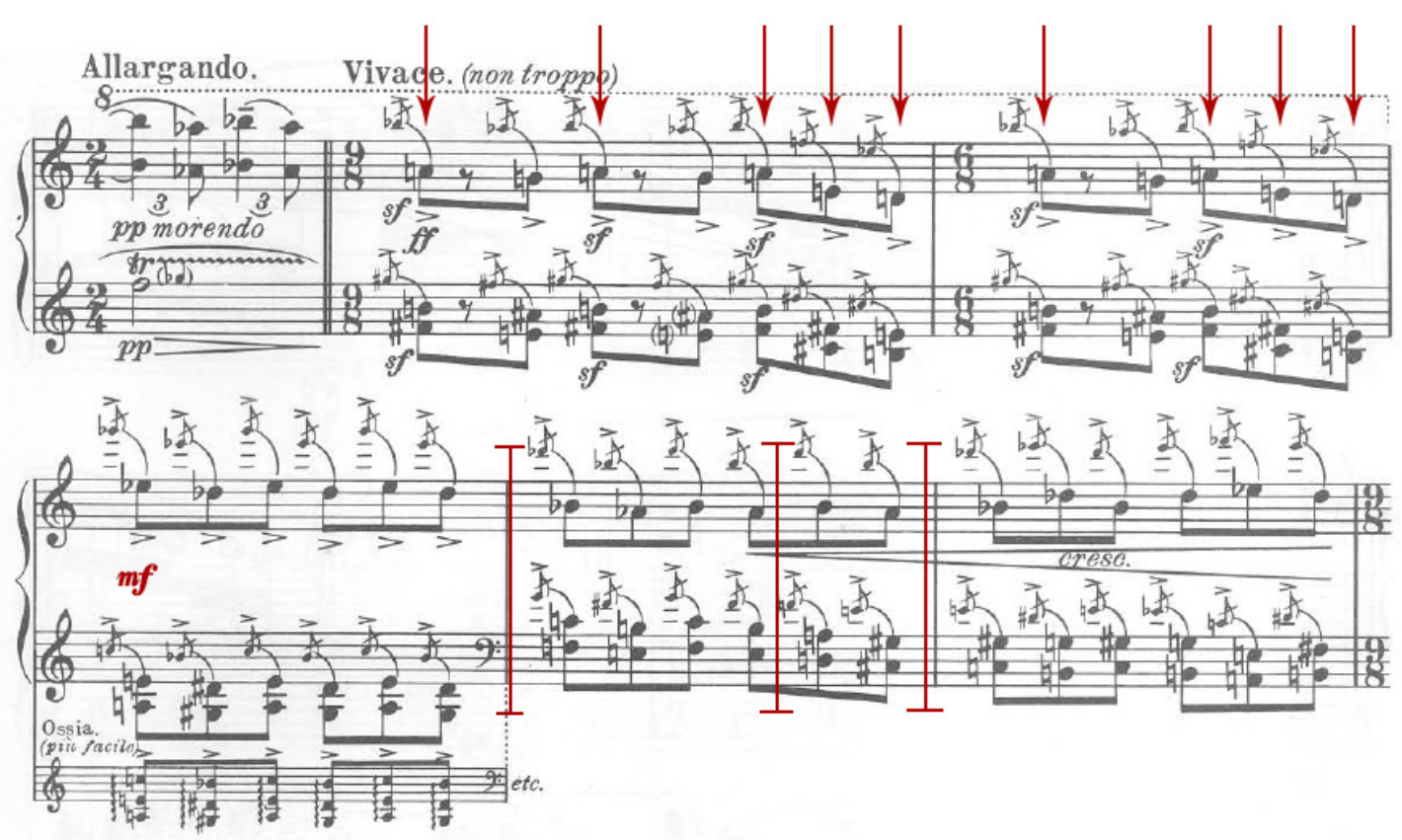

Figure 34 : Karol Szymanowski, Masques, op. 24 (1915-1916), "Tantris le bouffon» (n 2), mes. 53-58, indications interprétatives (accents, dynamique, respirations).

Plus loin, à la mesure 59, l'aboutissement du crescendo donne lieu à un brusque changement d'écriture dont la cassure se voit liée au rapport de demi-ton la-mi > lab-mib déployé à la main gauche et qu'il s'agit de souligner. La quinte lab-mib doit être posée par un geste de projection de l'avant-bras qui, par l'amplitude de son mouvement, va générer une légère distorsion du temps musical et déterminer le poids dans la frappe nécessaire à la perception d'une véritable intensité sonore. L'interprète procèdera de même à la mesure suivante pour manifester la relation de cohabitation quintale réb-lab.

Fruit de notre démonstration analytique précédente, l'implication de la double triade $f a \#$-la\#-réb/do-mi-sol issue des collections la\#-sol-fa\# et mi-réb-do, détermine plusieurs choix interprétatifs dans la section "Ancora meno » (mes. 47 et 101) et à la première mesure de la section "Allegro moderato » (mes. 24). Ainsi, dans cette dernière, les trois premiers temps suscitent à eux seuls la cohabitation tritonique d'une gamme de do majeur présentée à la main gauche sous la forme d'accords, et de la tierce affirmant la prégnance de l'univers de $f a$ dièse à la main droite. Ces trois premiers temps rassemblent également toute la problématique contenue dans la conclusion de l'œuvre : tandis que la main droite présente, par enharmonie, la tierce formant l'identité modale de l'accord final de sol bémol majeur, les trois accords de la main gauche montrent le geste cadentiel présenté à la main droite dans les trois dernières mesures de "Tantris », c'est-à-dire un accord de sol septième de dominante, suivi d'un accord $\mathrm{du} \mathrm{vI}^{\mathrm{e}}$ degré dans son second renversement. 


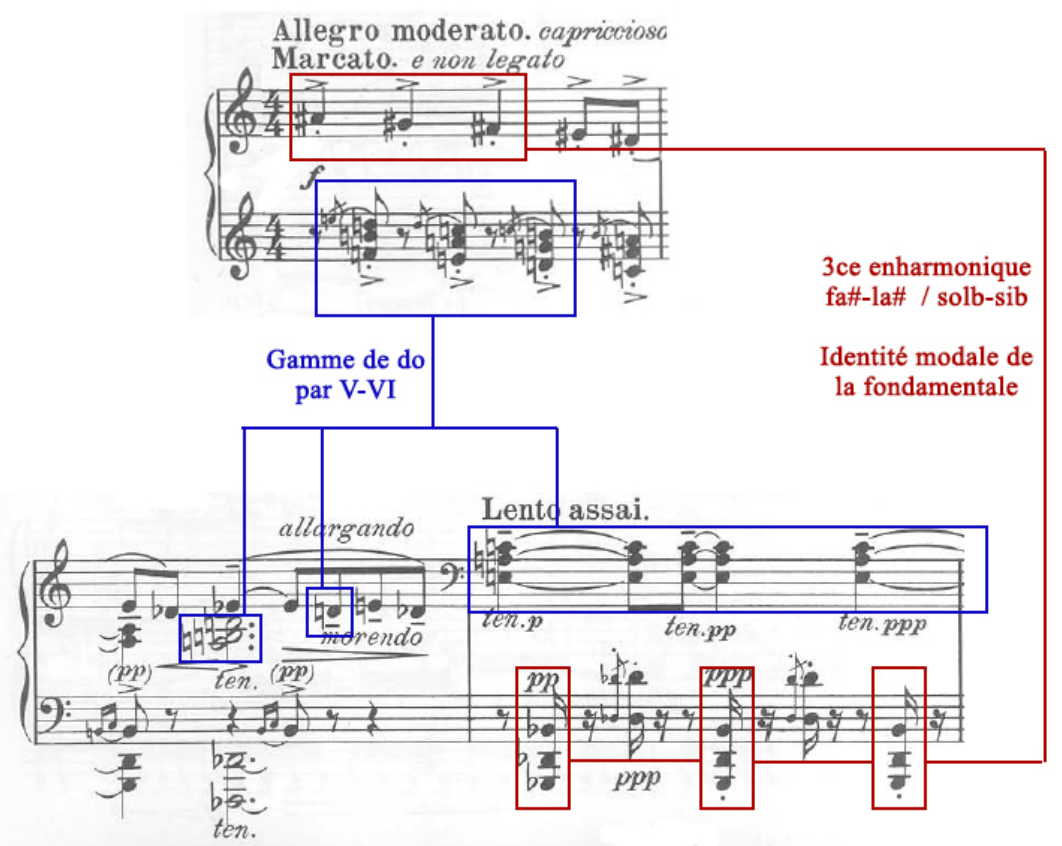

Figure 35 : Karol Szymanowski, Masques, op. 24 (1915-1916), "Tantris le bouffon" ( $\left.n^{\circ} 2\right)$, mes. 24 et 106-107, relations harmoniques.

Afin de signifier auditivement la valeur sonore de ce passage, l'interprète pourra énoncer avec un léger ralenti les deux premiers temps de la section (mes. 24), en marquant de façon égale la ligne mélodique à la main droite et les accords de la main gauche. Ceux-ci seront articulés dans un geste de projection de la main vers l'intérieur du clavier, le poignet se relevant à l'instant de la frappe de l'accord.

L'interprète averti de l'imprégnation de la double triade et de la relation tritonique fondamentale $f a \#-d o$ au sein de la section "Ancora meno " (mes. 47 et 101) sera en mesure d'investir son jeu pianistique d'une volonté expressive dont l'attention toute particulière sera portée au déploiement de la triade do-mi-sol à travers la cellule motivique à la main gauche. La réalisation de cette triade implique naturellement l'exécution de l'accentuel porté tout à la fois à la basse de l'accord (sol) et aux deux octaves $(m i, d o)$, ainsi qu'aux notes centrales blanches $(s o l, m i)$. Or, la production de l'accentuel à la main gauche par l'augmentation du poids d'enfoncement de la touche et l'allongement de la durée de la note, va contribuer spontanément à hiérarchiser, au sein de l'espace mélodique de la ligne supérieure de la main droite, les notes d'importance structurelles ( $f a \#-l a \#)$ de celle de passage (sol\#). 


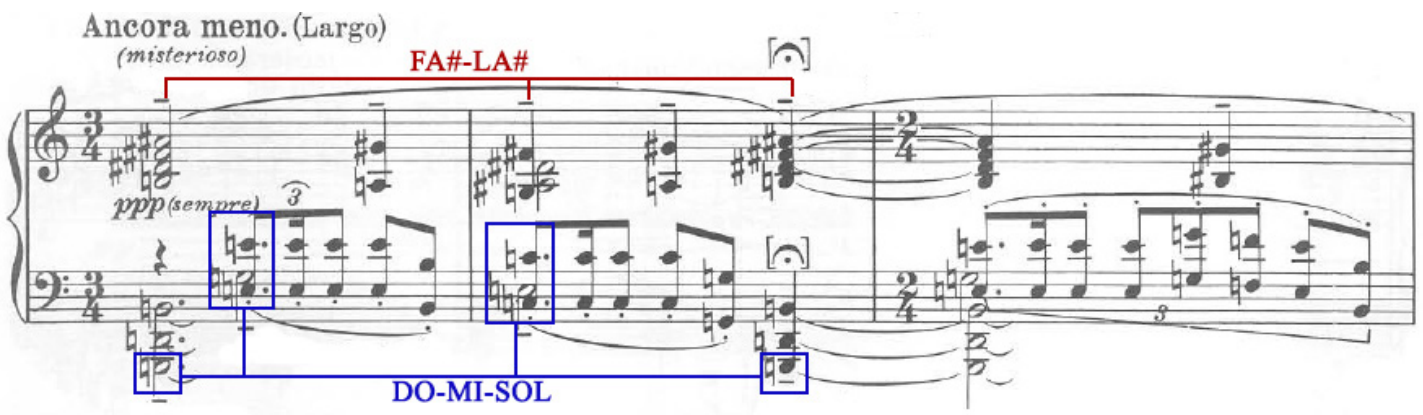

Figure 36 : Karol Szymanowski, Masques, op. 24 (1915-1916), "Tantris le bouffon» (n 2), mes. 47-49, impact de l'accentuel à la main gauche sur l'importance sonore des notes mélodiques de la main droite.

Enfin, aux mesures 82-85, l'espace musical caractérisé par une séparation bémol/bécarre, manifestation éclatante de la prégnance de la double triade, devra être énoncé en marquant bien les notes supérieures des tierces brisées à la main droite afin de rendre palpable le segment de gamme de do majeur et la tierce fondamentale de la triade : fa-mi-ré-do. Lorsqu'à la mesure 86 la gamme se complète de ses notes manquantes et qu'à la main gauche nous obtenons la fondamentale (sol bémol) du mode pentatonique, il faut que l'interprète savoure, en quelque sorte, la jonction des deux triades qui s'opère par le demi-ton solb-solf et qu'il retienne quelque peu le mouvement musical pour souligner ces deux notes.

Le complexe sonore qui fait suite à l'instant de cohabitation de la double triade montre un foisonnement d'indications d'accentuel et de dynamique desquelles il s'agit de distinguer un ordre de priorité expressive. Or, le relevé des notes marquées par les $s f$ présente, sur l'assise fondamentale si bécarre, une collection de trois notes qui résonnent immédiatement avec celles ouvrant la cellule motivique dans la section "Allegro moderato » (mes. 24), réitérée plusieurs fois au fil de l'œuvre, et, comme nous l'avons montré, dans les sections "Ancora meno » (mes. 47 et 101). Ainsi, lorsque l'on cherche à établir le parcours d'un dessin mélodique proéminent, on constate auditivement que les groupes de double et triples croches provoquent un élan qui conduit naturellement à l'affirmation des notes $s f$. À ce titre, l'interprète pourra choisir de mettre en valeur la ligne suivante : si-fa\#-la\#-sol\#-la\#-sol\#. En outre, de façon tout à fait remarquable, le sol dièse au deuxième temps se voit infléchi au sol bécarre puis au $\mathrm{fa}$ dièse : l'oscillation $\mathrm{sol}$ \#/sol $\mathrm{h}$, permettant un remplissage différent de la tierce fa\#-la\#, implique de facto la collection de trois notes dans la problématique de la double triade. L'interprète ménagera donc une légère inflexion du discours musical, donnant aux deux doubles croches sol-fa\#, par leur inclusion au sein du dessin mélodique, une expressivité soutenue. 

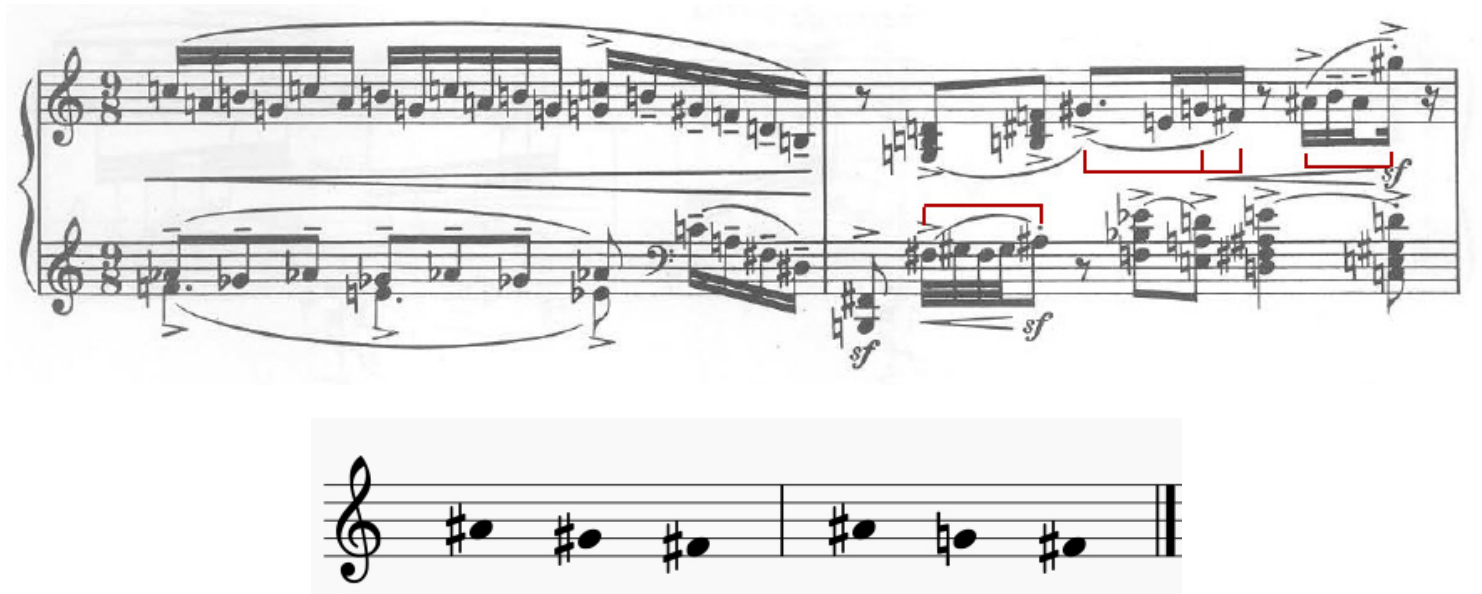

Figure 37 : Karol Szymanowski, Masques, op. 24 (1915-1916), «Tantris le bouffon» (n 2), mes. 85-86, présentation de la tierce fa \#-la \# et de son double remplissage.

Nous retrouvons ce segment de trois notes aux mesures 66 et 69 , inclus cette fois dans une réminiscence plus immédiatement perceptible de la cellule motivique issue de 1'«Allegro moderato » (mes. 24), réitérée deux fois et présentée d'abord à la main gauche, puis à la main droite, cette dernière se voyant transposée à la quinte et renouant avec sa tessiture originelle. Nous constatons également que les notes supérieures des quintes appuyées, à la main gauche, décrivent le même dessin mélodique, par enharmonie, que la première occurrence de la cellule, c'est-à-dire dans sa version altérée par une seconde mineure aux deux dernières croches. 

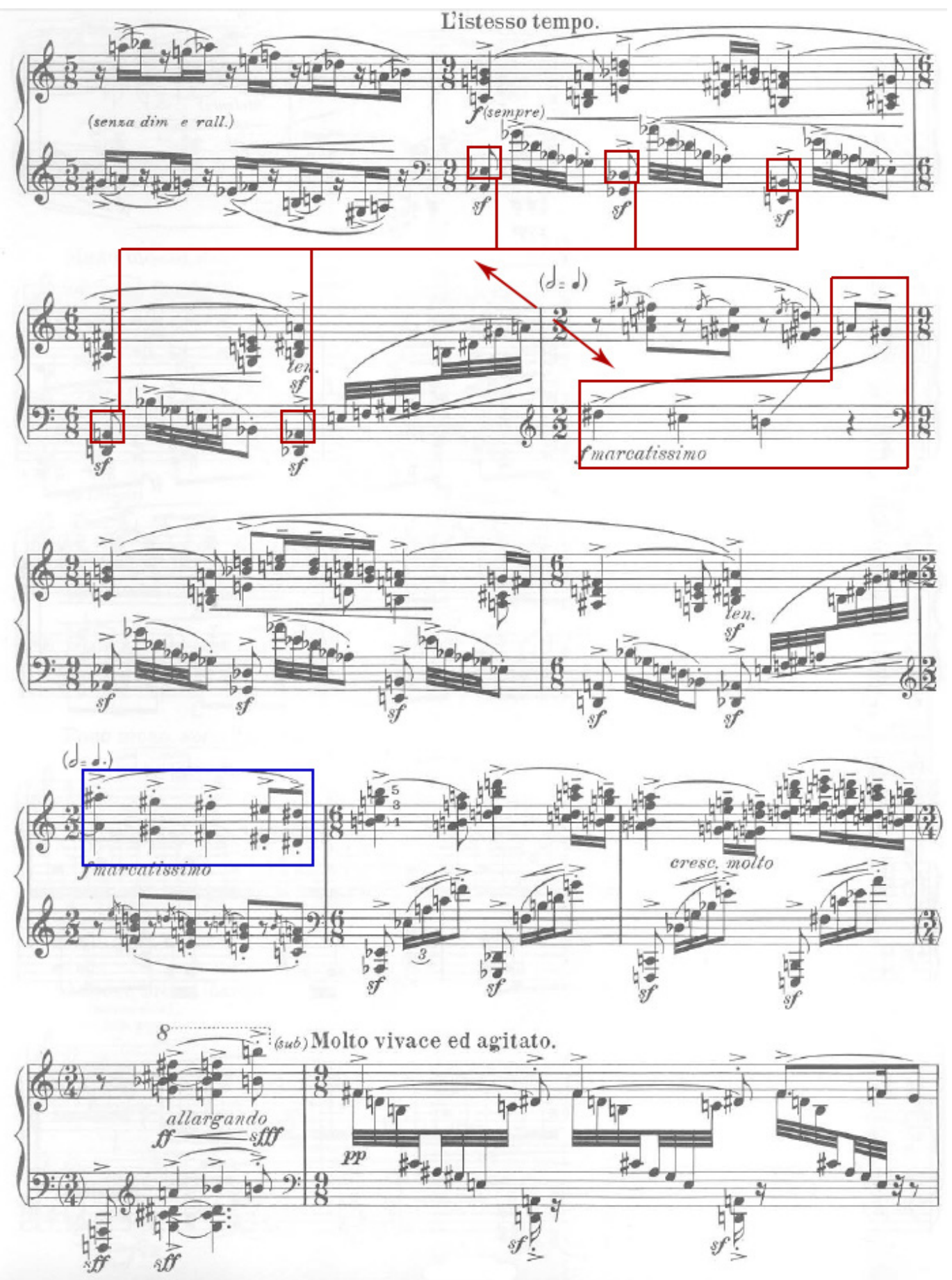

Figure 38 : Karol Szymanowski, Masques, op. 24 (1915-1916),

"Tantris le bouffon" ( $n^{\circ}$ 2), mes. 63-74, transformation de la cellule motivique.

Considérant à présent le signifiant intervallique déterminé par les extrémités des deux cellules motiviques, nous obtenons, pour la première, la quinte ré\#-sol\#, et pour la seconde, la quinte la ${ }_{-1}-r^{\prime} \sharp$, toutes deux ayant donc la capacité de former le segment de cycle suivant : sol\#-ré\#-la\#. Or, la conception intervallique de la première cellule par la survenance de la seconde mineure, ainsi que la prégnance du mouvement descendant décrit par les quintes aux deux mesures précédentes, tend à être assimilée 
auditivement comme centrée autour du pôle d'attraction do dièse, fondamentale d'une gamme décrivant le schème d'une oscillation modale appartenant au mode de mi et au mode de la.

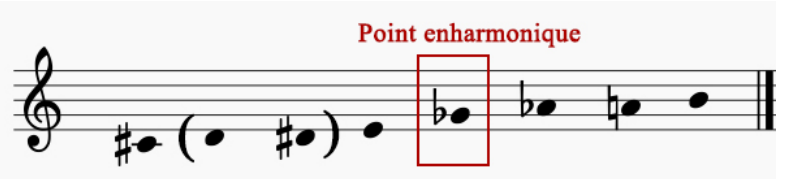

Figure 39 : Gamme obtenue, pouvant être rattachée au mode de mi et au mode de la.

Cette vue analytique, modelant la perception auditive, conduit à l'ajout de la note do dièse, fondamentale de notre gamme, au segment de cycle exposé plus haut, soit à présent : do\#-sol\#-ré\#-la\#. De plus, envisageant la seconde itération de la cellule motivique à l'aune de son implication fondamentale telle que présentée usuellement au cours de l'œuvre, nous y voyons décrit l'assise fondamentale sur fa dièse, note que nous adjoignons au bas du cycle. Le cycle ainsi obtenu, fa\#-do\#-sol\#-ré\#-la\#, présente la double ossature structurelle de l'œuvre, dont le la dièse figure non seulement l'élargissement, mais également l'identité expressive de la potentielle résolution de la neuvième $d o \#$-sol\#-ré\# vers la tierce fa\#-la\#.

Plus loin, le geste quintal présenté à la fin de la section (mes. 72) par le mouvement de la quinte ré-la vers sol, fondamentale d'un accord de septième de dominante doublement altéré, décrit un nouveau segment de cycle sol-ré-la. À celui-ci, nous pouvons adjoindre le signifiant harmonique porté par le déploiement de la gamme de do à la main gauche par l'intermédiaire d'accords accompagnant la deuxième cellule motivique. Ce nouveau cycle contient, comme nous le savons, les doubles altérations des quintes centrales de la double ossature structurelle. Or, si à présent, poussant jusqu'au bout le raisonnement analytique, nous tâchons d'imbriquer les deux cycles mis en lumière, en y incorporant également, et dans une logique de construction intervallique justifiée, les quintes descendantes caractéristiques à la main gauche, nous sommes en mesure de former un cycle de quintes complet qui a la particularité de montrer, sous quatre de ses constituants, une doublure enharmonique.

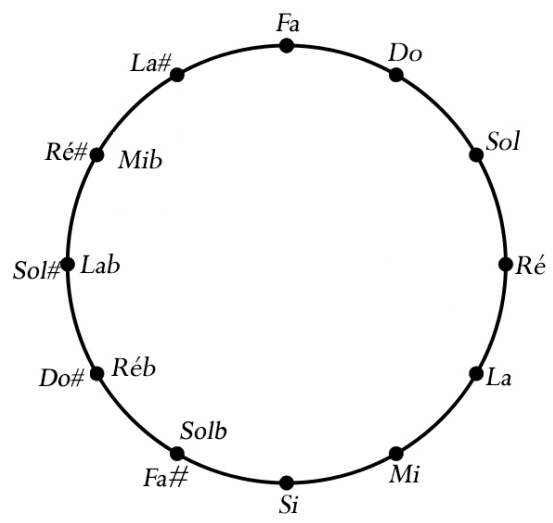

Figure 40: Cycle de quintes complet obtenu aux mes. 64-72 de "Tantris le bouffon» ( $\left.n^{\circ} 2\right)$ de Karol Szymanowski (Masques, op. 24, 1915-1916). 
S'il apparaît ici que la jonction enharmonique se réalise sur la quinte la\#-fa, la fin de la section (mes. 72) va permettre la complétion du cycle en bémol par la survenance du si bémol à la main gauche, au troisième temps de la dernière mesure. Entendue comme note de passage conduisant à la sensible de l'accord de dominante sur sol, elle n'en forme pas moins, avec le mouvement contraire de la ligne mélodique, la quinte sib-fa que l'interprète ne manquera pas de souligner à la mesure de l'augmentation dynamique et du ralentissement métrique au sein desquels elle se trouve englobée. À ce titre, nous assistons alors, dans notre cycle des quintes, à la cohabitation des cinq notes bémol/dièse, dont la base, le point de départ, est incarné par la note $f a \# / s o l b$. Or, cette note, dont l'implication enharmonique avait déjà été désignée dans les trois premières mesures de la section (mes. 64-66) par le choix d'écriture altératif de la gamme sur do dièse (voir figure 39), symbolise également le point tournant enharmonique du cycle décrit par les fondamentales de l'œuvre toute entière, tel que nous l'avons montré dans la figure 23.

\section{CONCLUSION/AU SUJET DU CYCLE DE QUINTES}

La démarche que nous adoptons de disposer les fondamentales relevées dans l'œuvre en cycle de quintes procède des propriétés internes du mode acoustique. Les notes non altérées forment une collection pentatonique (do-ré-mi-sol-la $\left.{ }^{13}\right)$ que nous disposons en cycle de quatre quintes successives : do-sol-ré-la-mi ${ }^{14}$. Or, si à partir de ce moyeu, nous élargissons le cycle à chacune des extrémités, nous obtenons les nouvelles notes si et $f a$, notes qui, ajoutées à la collection pentatonique, auraient la capacité de former le mode de do. Si nous poursuivons l'élargissement, nous obtenons alors, par échange des extrémités, les mêmes deux notes, altérées, qui fondent l'identité du mode acoustique, c'est-à-dire sa quarte haussée et sa septième abaissée : si bémol et $f a$ dièse.

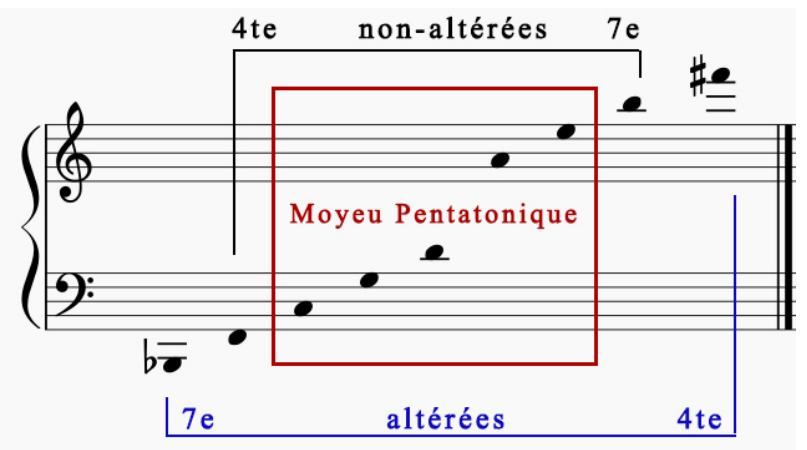

Figure 41 : Possibilité de former le mode de do, puis le mode acoustique, par élargissement du cycle des quintes à partir de la collection pentatonique.

13 Pour illustrer ce propos, nous déterminons que $0=d o$.

14 Voir à ce sujet McNamee 1985, p. 66. 
Ces deux notes altérées font basculer le cycle des quintes dans l'univers des " touches noires " (dièses ou bémols selon la notation enharmonique choisie). Le tétracorde 4-25 caractérisant le mode acoustique (do-fa\#/mi-sib) se voit doté de la capacité à former une double dyade (do-mi/solb-sib), dont chacune pourrait acquérir, selon le contexte musical, la capacité de devenir le point focal d'un système centré autour d'elle, c'est-à-dire de générer, à l'aune de la structuration globale, un geste décrivant une relation de trois notes à distances de quintes, associé de fait au principe de l'ossature quintale de neuvième décrit plus avant ${ }^{15}$.

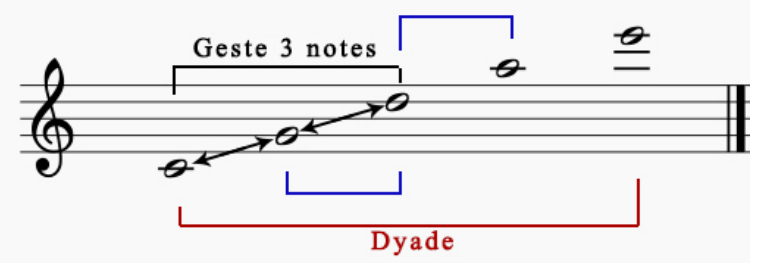

Figure 42 : Dyade do-mi pouvant générer un geste d'attractivité potentielle entre trois notes.

Le mode acoustique permet naturellement de mettre en œuvre, dans une disposition horizontale conjointe, une particularité intrinsèque du cycle des quintes : sa séparation médiane selon l'intervalle de triton. À ce titre, le mode acoustique permet un basculement aisé dans l'univers octotonique par la complétion d'un ou des deux cycles de tierces mineures. Dans l'optique d'une double dyade tritonique à caractère structurant, la complétion du cycle $C 3^{1}$ permet d'ajouter la quinte à la dyade solb-sib et ainsi de former, avec le sol originellement présent au mode acoustique, une double triade tritonique do-mi-sol/solb-sib-réb. Cette particularité de pouvoir former des accords parfaits, donc fondés sur la quinte à leurs extrémités, par l'interpénétration de cycles de tierces mineures, est une caractéristique propre à l'univers octotonique. Nous voyons par conséquent que l'usage du mode acoustique peut conduire à l'organisation de l'espace musical selon une pensée attachée à l'univers octotonique.

Notre démonstration théorique sur le mode acoustique nous emmène à diviser le cycle des quintes en deux groupes de quatre quintes formant deux collections pentatoniques à distance de triton : do-sol-ré-la-mi/solb-réb-lab-mib-sib. Cette présentation accrédite la thèse de l'acquisition d'une certaine dimension d'attractivité portée par les fondamentales de nos deux triades : les segments sol-ré-la et réb-lab-mib prenant rôle d'ossature à qualité dominante peuvent ainsi rejoindre les tierces do-mi et solb-sib, dans une formulation musicale souhaitant rappeler - et ce de façon peut-être sous-entendue - l'idiome tonal par l'accomplissement d'un geste intervallique de caractère résolutif.

15 Il s'agit de l'ensemble de classes de hauteurs 3-9, formé par la disposition du segment pentatonique non altéré du mode acoustique en deux ossatures de neuvième majeure (ex. : do-sol-ré + ré-la-mi, dans le cas d'un mode acoustique sur do). 

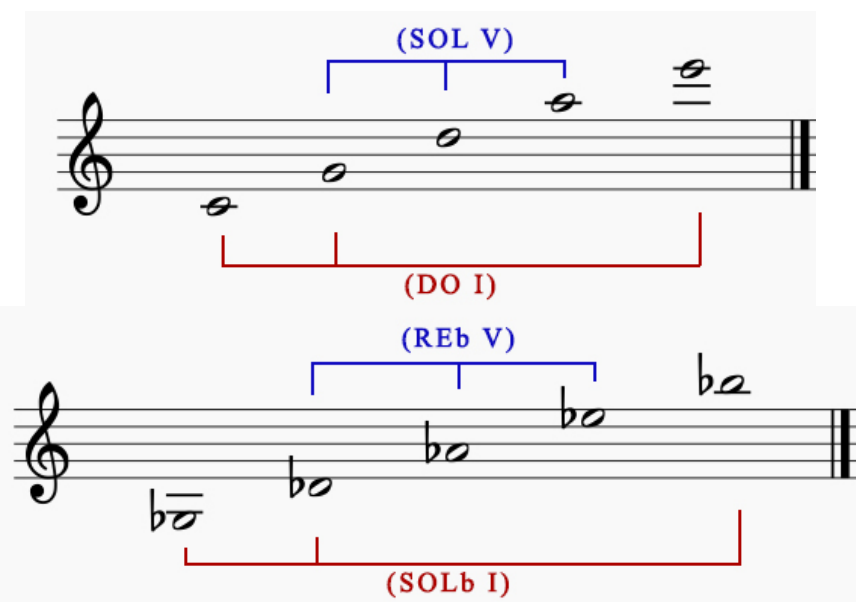

Figure 43 : Formulation résolutive possible au sein de l'échelle pentatonique.

Or, les notes manquantes à la complétion du cycle de quintes complet, c'est-à-dire $s i$ et $f a$ - notes altérées dans le mode acoustique - incarnent respectivement, dans une pensée héritée de la tonalité, les sensibles et septièmes dans les ossatures quintales citées ci-dessus et leur permettent d'acquérir, au sein de cet idiome, une véritable identité de dominante.

Lorsque l'on dispose en classes de hauteurs les notes qui constituent deux accords de neuvième de qualité dominante à distance de triton, on obtient une échelle de huit sons au sein de laquelle la notion de fondamentale dans la double ossature quintale se trouve de nouveau réévaluée. La nouvelle échelle formée (ensemble de classes de hauteurs 8-25) s'organise comme une collection symétrique dont le moyeu divise l'octave en son milieu exact par la CI6 qu'il forme avec les extrémités. Tandis que le triton détermine la structuration de l'échelle, celle-ci permet également l'implication de la quinte comme centricité tonale, par la capacité qu'elle possède de constituer deux agrégats altérés de neuvième de qualité dominante - dont les fondamentales, à distance de triton, marquent les extrémités des deux accords originels - et qui disposent de la possibilité de se résoudre par mouvement descendant vers un accord parfait mineur constitutif de l'échelle. 

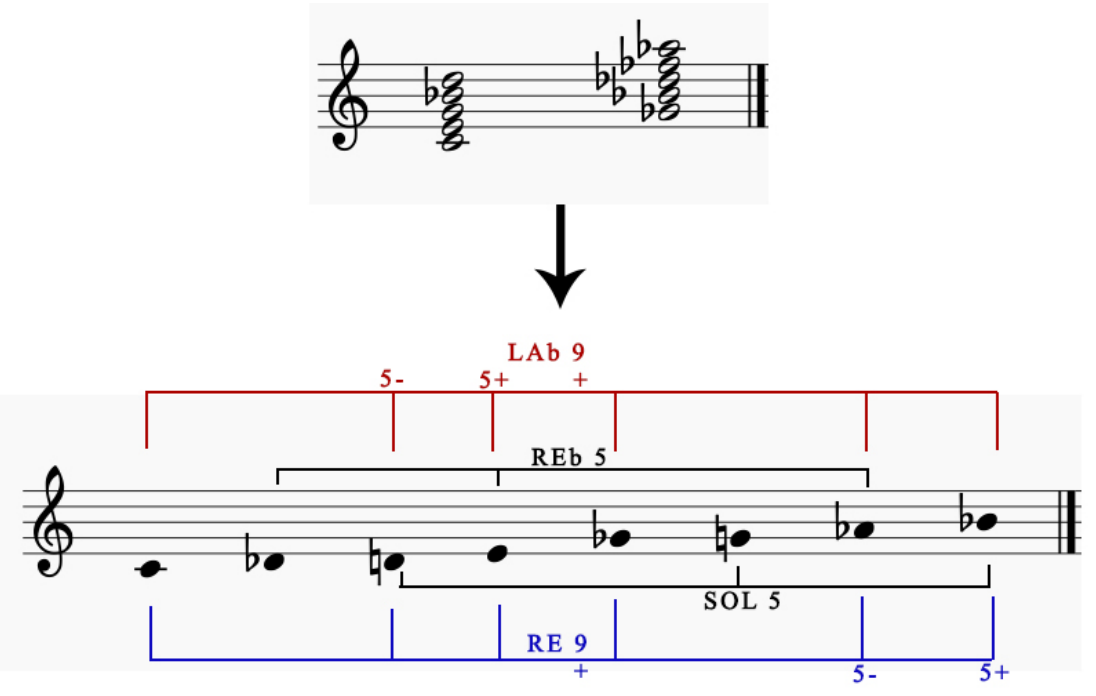

Figure 44 : Deux accords de neuvième altérés de qualité dominante et leur capacité résolutive vers deux accords parfaits mineurs.

Cette double relation quintale - produite originellement par l'ossature de neuvième majeure - se déterminant par son habillage harmonique, est détachée de la pensée tonale par le fait qu'aucune note du cycle des quintes n'agit comme un pôle d'attractivité univoque, bien qu'il soit tout de même possible d'y voir scintiller une certaine forme de réminiscence des principes fondateurs de cet idiome par la coloration de fondamentales en qualité dominante et les déplacements de quinte qu'elles réalisent ensuite. L'exemple suivant, tiré de " Tantris le bouffon ", est révélateur à ce titre de la mise en œuvre de ce système d'organisation. 

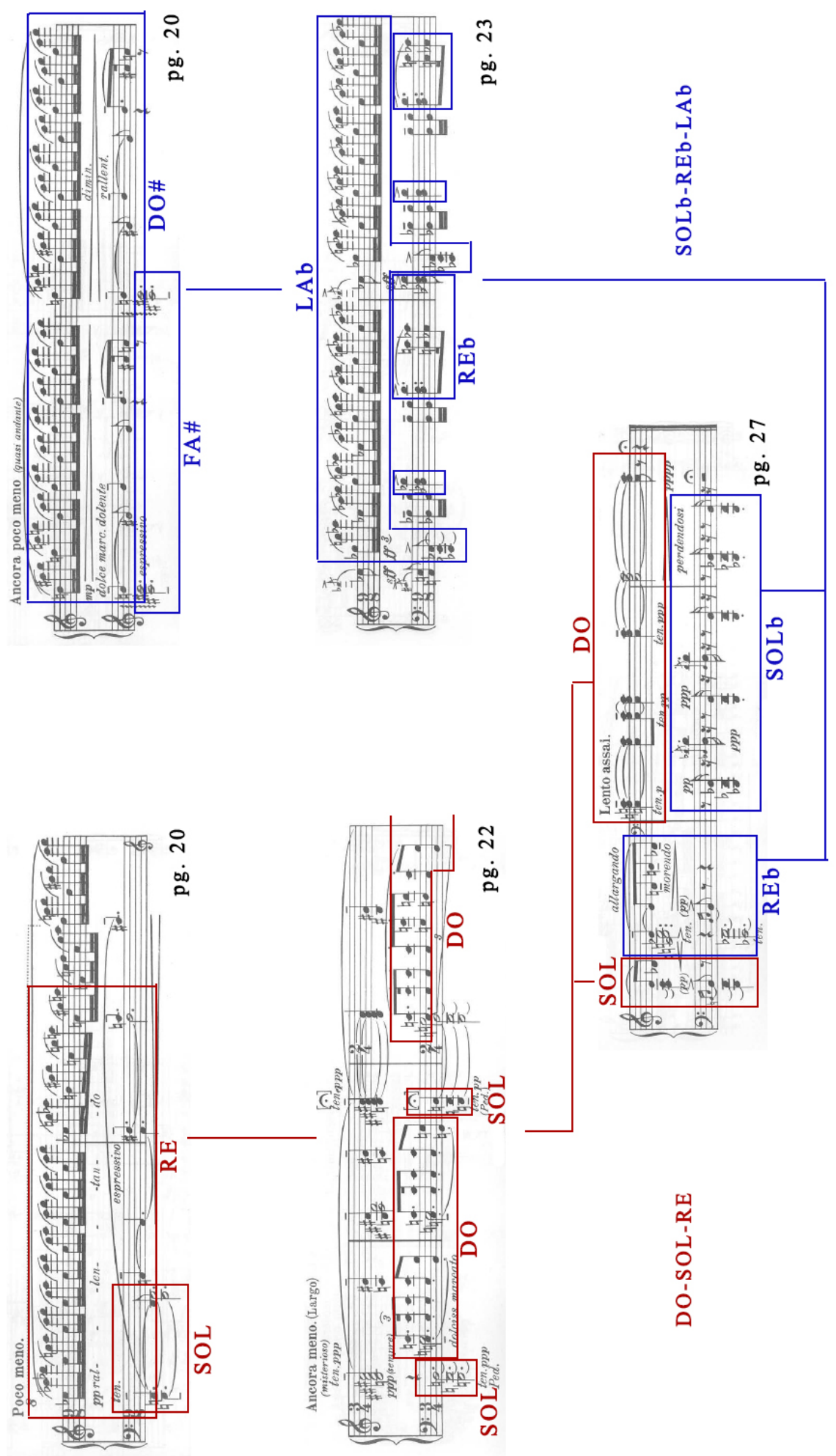

Figure 45 : Implication principale de la double ossature quintale à distance de triton au sein de "Tantris le bouffon» ( $n^{\circ}$ 2) de Karol Szymanowski (Masques, op. 24, 1915-1916) ${ }^{16}$. 


\section{BIBLIOGRAPHIE}

Antokoletz, Elliott (2014), A History of Twentieth-Century Music in a Theoretic-Analytical Context, New York/Londres, Routledge.

Awakawa-Imai, Tchié (2009), " "Tantris le bouffon" (extrait des Masques) de Karol Szymanowski. Un exemple d'ekphrasis musical inspiré par la pièce de théâtre d'Ernst Hardt ", dans Bérangère Mauduit (dir.), Dramaturgie et musique. Actes de la rencontre interartistique du 22 mars 2006, $\mathrm{CS} \mathrm{n}^{\circ} 39$, p. 61-72.

Barraqué, Jean (1962), Debussy, Paris, Seuil.

Berger, Arthur (1963), "Problems of Pitch Organization in Stravinsky ", Perspectives of New Music, vol. $2, \mathrm{n}^{\circ} 1$, p. 11-42.

Chechlińska, Zofia (1973), "Chopin a impresjonizm » [ Chopin et l'impressionnisme »], dans Zofia Chechlińska (dir.), Skice o kulture muzycznej XIX wieku. Studia i materialy, tome 2, Varsovie, Państwowe Wydwanictwo Naukowe, p. 21-34.

Chylińska, Teresa (1973), Szymanowski, New York, Twayne Publishers \& The Kosciuszko Foundation.

Chylińska, Teresa (1982), Korespondencja, "Vol. 1. 1903-1919 », Cracovie, Polskie Wydawnictwo Muzyczne.

Chylińska, Teresa (1993), Karol Szymanowski. His Life and Works, traduit par John Glowacki, Alderhost, University of Southern California.

Chylińska, Teresa (2006), Karol Szymanowski i jego epoka, Cracovie, Musica Iagellonica.

Durval, Cesetti (2012), "The Many Masks of Karol Szymanowski. A Discussion of his Two Piano Triptychs », Revista Musica Hodie, vol. 12, no 1, p. 117-137, www.revistas.ufg.br/musica/article/ view/20231/12643, consulté le 3 octobre 2016.

Fletcher, Marylynn Louise (1984), Pitches Constructions in the Masques, op. 34 of Karol Szymanowski, Thèse de doctorat, Université du Texas.

Kurth, Ernst (1920), Romantische Harmonik und ihre Krise in Wagners "Tristan", Bern, P. Haupt, https://archive.org/details/romantischeharmo00kurt, consulté le 3 octobre 2016.

Leleu, Jean-Louis (1995), "La notion de "Background Structure" chez George Perle. De l'étude du langage musical au déchiffrement des œuvres ", International Journal of Musicology, vol. 4, p. $253-290$.

Leleu, Jean-Louis (2004), «Structures d'intervalles et organisation formelle chez Debussy. Une lecture de "Sirènes" ", dans Maxime Joos (dir.), Claude Debussy. Jeux de formes, Paris, Éditions Rue d’Ulm (ENS), p. 189-219, 288-95.

Leleu, Jean-Louis (2013), « Debussy selon Ernst Kurth. La mise en perspective du théoricien », dans Myriam Chimènes et Alexandra Laederich (dir.), Regards sur Debussy, Paris, Fayard.

Łobaczewska, Stefania (1950), Karol Szymanowski, Zycie i twórczość, Cracovie, Polskie Wydawnictwo Muzyczne.

McNamee, Ann K. (1985), "Bitonality, Mode, and Interval in the Music of Karol Szymanowski », Journal of Music Theory, vol. 29, $\mathrm{n}^{\circ}$ 1, p. 61-84.

Messiaen, Olivier (1966), Technique de mon langage musical, Paris, Leduc.

Moere van, Didier (2008), Karol Szymanowski, Paris, Fayard.

Palmer, Christopher (1987), Szymanowski, un compositeur à la croisée des chemins, traduit par M. Tchamitchian-Faure, Paris, Actes Sud.

Perle, George (1977), Twelve-Tone Tonality, Berkeley/Los Angeles, University of California Press.

Perle, George ([1990]1996), The Listening Composer, Berkeley/Los Angeles, University of California Press. 
Riemann, Hugo (1901), "Zur Theorie der Konsonanz und Dissonanz », Präludien und Studien. Gesammelte Aufsätze zur Asthetik, Theorie un Geschichte der Musik, vol. 3, Leipzig, H. Seemann, p. 31-46.

Samson, Jim (1990), The Music of Szymanowski, Londres, Kahn \& Averill.

Sierpiński, Zygmunt (1983), O Karolu Szymanowskim, Varsovie, Interpress.

Stumpf, Carl (1898), "Konsonanz und Dissonanz », Beitrage zur Akustik und Musikwissenschaft, vol. 1, p. 1-108, http://echo.mpiwg-berlin.mpg.de/MPIWG:8UEXS5PM, consulté le 3 octobre 2016.

Stumpf, Carl (1911), "Konsonanz und Konkordanz ", Beitrage zur Akustik und Musikwissenschaft, vol. 6, p. 116-150, http://echo.mpiwg-berlin.mpg.de/MPIWG:HZGZZ966, consulté le 3 octobre 2016.

Wightman, Alistair (1999), Karol Szymanowski. His Life and Work, Alderhost, Ashgate Publishing.

Wightman, Alistair (1999), Selected Writings of Szymanowski, Londres, Toccata Press.

Zent, Donald (1988), The Harmonic Language of Karol Szymanowski's "Métopes," op. 29, and "Masques," op. 34, Université de Cincinnati.

Zieliński, Tadeusz (1997), Szymanowski, Liryka i ekstaza, Cracovie, Polskie Wydawnictwo Muzyczne. 